\title{
A study of the causal relationship between the emergence of a twisted magnetic flux rope and a small $\mathrm{H} \alpha$ two-ribbon flare
}

\author{
D. H. Brooks ${ }^{1}$, H. Kurokawa ${ }^{1}$, K. Yoshimura ${ }^{1,2}$, H. Kozu ${ }^{1}$, and T. E. Berger ${ }^{3}$ \\ ${ }^{1}$ Kwasan and Hida Observatories, Kyoto University, Yamashinaku, Kyoto 607-8471, Japan \\ 2 Institute of Space and Astronautical Sciences, Sagamihara, Kanagawa 229-8510, Japan \\ ${ }^{3}$ Lockheed Martin Solar and Astrophysics Lab, O/L9-41, B/252, 3251 Hannover Street, Palo Alto, CA 94304, USA
}

Received 9 December 2002 / Accepted 19 August 2003

\begin{abstract}
We present results from an analysis of a small two-ribbon flare which occurred above emerging flux in solar active region NOAA 8218 on 1998, May 13th and which was observed by the Swedish Vacuum Solar Telescope (SVST) on the island of La Palma, Spain. The relatively simple magnetic morphology and small size of the flare together with the high quality of the SVST observations allow us to examine the essential properties of flares in emerging flux regions in greater detail than before. In this paper we compare and contrast the flaring emerging flux region simultaneously with a non-flaring emerging flux region within the same field of view. Unusual magnetic footpoint motions are observed in the flaring region, coincident with the $\mathrm{H} \alpha$ kernels, which result in a high level of shearing of the magnetic neutral line between opposite polarities. The $\mathrm{H} \alpha$ images show dark filament structures which form an inverted S-like shape immediately prior to the flare and then separate after the energy release disrupts the magnetic field. We interpret the motions and structures as strong evidence for the emergence of a twisted magnetic flux rope which developed a sheared configuration with the overlying magnetic field. In contrast the companion region shows separating footpoints, with apparent arch-like filament connections in the $\mathrm{H} \alpha$ images, consistent with the expected appearance of emerging flux. The observations imply that the attachment of the inverted S-shaped structure may be an observational consequence of the magnetic reconnection or untwisting of the field which triggered the flare. We also find some evidence that the increase in magnetic flux is faster in the flaring region.

Finally, we propose a simple schematic model of the emergence of a twisted magnetic flux rope and attached branch which can account for the observed footpoint motions and $\mathrm{H} \alpha$ structures of the flaring region. Such a model can, in principle, induce partial magnetic reconnection in the overlying coronal field and we found some evidence of coronal loop footpoint brightenings which support our conclusions. Our high resolution study supports the results of previous authors that even a small twisted structure in an emerging flux tube can be important for flare production.
\end{abstract}

Key words. Sun: photosphere - Sun: chromosphere - Sun: corona - Sun: magnetic fields - Sun: flares

\section{Introduction}

The causal link between magnetic flux emergence from below the photosphere and the generation of solar flares is a topic of much theoretical and observational interest. Since the gas pressure exceeds the magnetic pressure in the convection zone twisting and rolling up of the field lines must occur. As the flux emerges above the photosphere magnetic shear can occur due to the subsurface motions and this allows the build up of energy before a flare (Zirin 1983). From a theoretical point of view it is expected that the twist of the magnetic field lines will relax to a lower magnetic configuration as the gas pressure decreases. It has been suggested that this relaxation of the twisted magnetic field, either through reconnection with the preexisting coronal field and/or through untwisting, releases the stored magnetic energy in the form of solar flares (Piddington 1974;

Send offprint requests to: D. H. Brooks, e-mail: dbrooks@kwasan.kyoto-u.ac.jp van Driel-Gesztelyi et al. 1996; Ishii et al. 1998). To date, however, the precise physical mechanism by which a flare can be triggered is unconfirmed and still the subject of much continuing effort and debate.

Heyvaerts et al. (1977) proposed a scenario in which emerging magnetic loops interact with the overlying coronal magnetic field resulting in energy release through magnetic reconnection in the current sheet which forms between the old and new flux. Differences in flare types could then be explained by the complexity of the corresponding coronal fields into which the new magnetic flux emerges. Since then many authors have further developed aspects of the emerging flux reconnection models or interpreted ground and space based observations within this model framework (for just a few examples see Forbes \& Priest 1984; Kurokawa 1991; Shibata et al. 1992; Linton et al. 2001; Ishii et al. 2000; Li et al. 2000; Ranns et al. 2000). 
The importance of magnetic shear has been stressed on many occasions (Zirin \& Tanaka 1973; Hagyard et al. 1984; Kurokawa 1987; Tanaka 1991; Kurokawa 1996; Ishii et al. 1998). Some of these authors have focused on the twist of the rising flux tubes as the essential element in flare production. For example, Kurokawa (1987) showed that the successive emergence of twisted magnetic loops can lead to the rapid development of magnetic shear and subsequently strong flares. Tanaka (1991) suggested that the emergence of twisted magnetic flux could explain the increase and subsequent decrease in magnetic shear associated with spot growth and decay in a region of intense flares he observed. Ishii et al. (1998) interpreted unusual vortex-like sunspot motions as evidence for the emergence of twisted magnetic flux and shear development and found that the strongest flares occurred at the site of the vortex-like motions. More recently, Kurokawa et al. (2002) successfully constructed a realistic model of a strongly twisted flux rope to explain the drastic changes in an S-type sunspot configuration which produced strong flare activity. Li et al. (2000) studied $\mathrm{H} \alpha$ and soft $\mathrm{X}$-ray observations of a number of flares and suggested that emerging flux was an important factor in flare occurrence in regions of low magnetic shear.

Many of these authors studied the typical geometry of flaring flux tubes as this can be important for predicting flare occurrence (Ishii et al. 1998). However, many of the studies were based on observations of quite complex structures and motions in evolving regions. Therefore, studies of flares in apparently simple geometries should additionally be very useful for understanding more clearly the evolutional characteristics of emerging magnetic flux and for clarifying the relative importance of twisting and/or shearing of the magnetic field in flare production.

Here we present results from an analysis of a small tworibbon flare which occurred in solar active region NOAA 8218 on 1998, May 13th. The region was observed by the $50 \mathrm{~cm}$ Swedish Vacuum Solar Telescope (SVST), formerly located on the island of La Palma, Spain (the telescope has recently been upgraded to the 1-m class New Swedish Solar Telescope). The data provide a good opportunity to study the causal relationship between magnetic flux emergence and solar flares. The high spatial resolution (close to $0.3^{\prime \prime}$ ) and coverage of the SVST observations simultaneously at several wavelengths over 3 hours and $50 \mathrm{~min}$, allow us to examine in great detail the temporal evolution of the characteristics and structure of the region and associated magnetic field throughout the flare duration. The observations were supplemented with low resolution full disk data from SOHO/MDI. Full-disk images from SOHO/EIT which partially overlapped with the SVST observing period, were also examined.

The active region contains two large sunspots around which we identified considerable activity; some pore movements, numerous brightenings, transient tube like structures and the small two-ribbon flare. Unusual magnetic footpoint motions are observed, coincident with the $\mathrm{H} \alpha$ flare kernels, which result in a high level of shearing of the magnetic neutral line. We interpret the motions as strong evidence for the emergence of twisted magnetic flux. We study the temporal evolution of the structure of the flare and the associated magnetic field. The
$\mathrm{H} \alpha$ images show two dark structures which form an inverted S-like shape immediately prior to the flare. We find that the flare occurs during a period of attachment and then separation of these structures. The Fe I data has been examined to build up a picture of the emergence and reconfiguration of the associated magnetic field. We also simultaneously study a nearby non-flaring emerging flux region, within the same field of view (FOV), in order to determine the essential difference between the two regions. This difference is likely to be related to the flare trigger, the mechanism and location of which are still uncertain.

In the next section we briefly discuss the SVST observations and the general data processing which they required. In Sect. 3 we present a complete analysis of the small two-ribbon flare. In Sect. 4 we interpret the results within the framework of an emerging twisted magnetic flux model and discuss the results. In Sect. 5 we finish with a short summary.

\section{Observations}

The observations used in this work come principally from the Swedish $50 \mathrm{~cm}$ vacuum solar telescope situated on the island of La Palma, Spain. The conceptual design, instrumentation and capabilities are given by Scharmer et al. (1985) and Scharmer \& Lofdahl (1991). The data were complemented with solar full disk images from SOHO-EIT in the Fe XII $\sim 195 \AA$ line and additionally full disk magnetograms from SOHO-MDI. The details of these instruments are given by Delaboudinière et al. (1995) and Scherrer et al. (1995).

\subsection{The 13th May 1998 dataset}

Solar Active Region NOAA 8218 (S20 W20) was observed on 1998, May 13th between 12:44 and 16:38 UT by the SVST in the Ca II $3933 \AA$ K-line, the $4305 \AA G$-band and $\mathrm{H} \alpha$ (6356 A). Simultaneous magnetograms were obtained using the Fe I 6302 A line. Data were obtained by the Lockheed Solar Optical Universal Polarimeter (SOUP) tunable birefringent filter and the $G$-band and $\mathrm{Ca}$ II K-line interference filter systems (for a recent discussion of these instruments see Berger \& Title 2001).

After an initial scan of the Fe I $6302 \AA$ magnetogram pair ( $-60 \mathrm{m \AA}$ left and right hand circular polarisation components) and continuum wavelengths $(-350 \mathrm{~m} \AA$ ) with the tunable filter, the $\mathrm{H} \alpha(\sim 6356 \AA)$ bandpass was scanned sequentially at -700 , $-350,0,+350$ and $+700 \mathrm{~m} \AA$. The tunable filter was slightly defocused in $\mathrm{H} \alpha$ to accommodate the magnetogram pairs which were also very slightly defocused and obtained immediately following the $\mathrm{H} \alpha$ scan. Thus the sequence was completed with a scan of Fe I. The sequences used were MGHASCAN2.SEQ and HASHORT2. The $\mathrm{H} \alpha$ and $\mathrm{Fe}$ I data full fields-of-view were 2.13 by 1.43 arcmin $(1520 \times 1024$ pixels $)$. The best observational cadence for the $\mathrm{H} \alpha$ and $\mathrm{Fe}$ I datasets was $\sim 126.6 \mathrm{~s}$ with values ranging from this up to $\sim 155 \mathrm{~s}$. Over 90 images were obtained for each wavelength.

Continuous Ca II $3933 \AA$ K-line and $G$-band ( 4305 $\AA$ ) filtergrams were obtained over the same observing period as the tunable filter. The Ca II $3933 \AA$ K-line data have been studied 
but are not central to the conclusions of this work so are not discussed further in this article. The best observational cadence for the $G$-band images was $\sim 4.5 \mathrm{~s}$ with values ranging up to $\sim 58 \mathrm{~s}$. In all 441 images were recorded. The field-of-view was larger than the tunable filters: 2.82 by $2.82 \operatorname{arcmin}(2000 \times 2000$ pixels).

NOAA 8218 was not specifically targetted by either the EIT or MDI. However, full disk images and magnetograms are available from the respective data archives. The MDI magnetograms were obtained at one minute intervals from 12:00:0412:33:04 UT, 14:58:04-15:07:04, 15:09:04-15:59:04 UT and 16:01:04-16:59:04 UT. EIT images were obtained between 12:50:54 and 16:30:35 UT at approximately $31 / 2$ to $35 \mathrm{~min}$ intervals. In some cases the time coincidence was quite good (e.g. 1.9 s) but due to the MDI data coverage gap between $\sim 12: 30$ and 15:00 UT there are some differences of over an hour.

The MDI data were registered with the SVST magnetograms (see next section) to allow identification of the positioning of EIT features in the SVST dataset. The time coincidence of the MDI and SVST magnetograms was $22 \mathrm{~min}$ and $12 \mathrm{~s}$ on average reaching $0.1 \mathrm{~s}$ at best with differences of over an hour again caused by the MDI observations gap.

\subsection{General data processing}

The SVST uses an altitude azimuth mount which causes the solar image to rotate continuously throughout the observing period. Therefore it is necessary to derotate all the images. For comparison with EIT and MDI and for presentation of results we subsequently rotated selected images so that solar North is up and East is to the left. Nearly time coincident MDI and SVST data were used for this purpose and registered by comparison of features common to the two magnetograms. We also coaligned the individual image arrays for the production of movies for feature analysis. This was achieved by obtaining the relative $x / y$ pixel offsets for each image through a crosscorrelation method and subsequently aligning them using bilinear interpolation.

$G$-band and tunable filter images are obtained through different optical systems so before shifting the images to the same coordinate frame and further cross-alignment it is necessary to rescale them. Although $G$-band and $\mathrm{Fe}$ I images show the most common features (high resolution umbral structure for example) the main purpose of the Fe I data was the production of magnetograms. As with the $G$-band and K-line datasets they were coaligned, but the process was less critical and so following the others $\mathrm{H} \alpha+350 \mathrm{~m} \AA$ was used (see below). This is in any case an easier process for the Fe I data since they were obtained through the tunable filter and therefore the same optical system as $\mathrm{H} \alpha+350 \mathrm{~m} \AA$. However, where a substantial improvement was possible, i.e. in the coalignment of the magnetograms, the $G$-band and Fe I data were used (see below).

Observable bright points and features in the $G$-band and $\mathrm{H} \alpha$ datasets do not necessarily correspond to the same underlying structures so the rescaling was made with principle attention paid to large magnetic features such as sunspots. On occasion, when bright structures were clearly identifiable in both images, they would be used. A number of procedures were followed for this purpose.

To illustrate the methods consider the $G$-band images which we rescaled to the $\mathrm{H} \alpha+350 \mathrm{~m} \AA$ data. Firstly, a 1.40 by 1.40 arcmin subregion of the $\mathrm{H} \alpha+350 \mathrm{~m} \AA$ images around the area of interest and common to all wavelength datasets was selected. The coalignment method we used required the images to have the same size and the rebinned $\mathrm{H} \alpha$ data represented a good compromise between cutting down the image size for memory constraints and retaining resolution for visual inspection. Following this step, all time-coincident images were selected (a total of 6 in this case). This was done in order to take some account of possible degradation due to seeing and differences over the observing period. Then a "first guess" of the scaling factor between each of them was obtained by comparison of several pairs of cursor clicks on the same locations in the images. Then an image registration routine (IMAGE_REGISTER.PRO in SolarSoft) was used to determine the relative scaling between images. The procedure determines the affine transformation parameters of an image onto a reference image using least squares estimation (see Jähne 1997). The calculated scalings from the image registration and the cursor clicks were then compared to build up an array of around 21 scaling factors (to three significant figures) which adequately covered the range of values found for each timecoincident image pair. The images to be registered were then scaled by all these factors and the resultant array compared to the reference image visually using IDL graphics as an aid. The best scaling factor for each reference image was selected and finally an average of these was taken to decide a scaling factor for all the images. For these data the best scaling factors for the individual image pairs covering the full observation period varied less than $2 \%$ from the chosen final scaling factor. Since the $\mathrm{H} \alpha$ and $\mathrm{Fe}$ I data were recorded through the same tunable filter there was not expected to be any difference in scaling. However, to confirm this we followed the same analysis path and found that in the worst case the scaling factor between the different wavelength datasets was 1.008 .

All the $G$-band and Fe I images were then rescaled according to these factors. An appropriate center point in each wavelength dataset was chosen for the rescaling to ensure that the expanded images included the $\mathrm{H} \alpha$ area of interest after they had been resized for coalignment. The coalignment was then carried out using the procedure discussed above. In a final step, all the data were inspected to remove any poor quality images.

The MDI magnetograms and EIT images were coaligned making use of procedures from the SOHO/EIT team and D. Zarro's IDL mapping software, both available in SolarSoft. As mentioned above the comparison with the SVST dataset was made through registration of SVST and MDI magnetograms. The coalignment of the SVST data to the MDI images was not as accurate as the respective ground and space data internal coalignment. Since we used the MDI and SVST magnetograms for registration the lack of resolution from MDI prevented us from precise small scale feature matching. However, having determined the rotation and scaling (horizontal and vertical) factors between the magnetograms, we registered the coaligned processed magnetograms to assess the accuracy of the method. 
We found that the residual uncorrected rotation and scaling factors produced a difference of less than $2 \%$ from the values that were adopted. Such residuals are not sufficient to alter the conclusions of our study (see below).

SVST magnetograms were obtained from the Fe I left hand circular polarisation (LCP) and right hand circular polarisation (RCP) component datasets following the same methods as Berger \& Title (2001) and Berger \& Lites (2002). That is, the LCP and RCP datasets were corrected for flat-fielding, dark current effects and rotation. They were subsequently coaligned, their rigid displacements measured and the RCP images were destretched onto the LCP images. Such a procedure compensates partially for distortions in the images due to changing seeing conditions between the different observation times. Note that each LCP and RCP pair are separated by a minimum of around $13 \mathrm{~s}$. A final coalignment was made and a visual inspection confirmed the methods were effective. As discussed above, the Fe I and $G$-band images show many common features and alignment between them was judged to be superior to alignment between the magnetograms and the $G$-band images. Therefore, this coalignment was made prior to calculating the magnetograms. Since the $G$-band data had already been coaligned with the tunable filter data the magnetograms were automatically pre-aligned to the $\mathrm{H} \alpha+350 \mathrm{~m} \AA$ images.

Berger \& Lites (2002) describe the details of magnetogram calibration from SOUP filter data and the reader is referred there for a thorough discussion. After calibration some magnetograms were found to be of reduced quality due to seeing or blurring problems in one or other of the Fe I LCP or RCP images. These were rejected from the subsequent analysis. In addition, the procedure for calibrating the magnetograms does not provide information on the magnetic field strength above $1800 \mathrm{G}$ so we applied the condition $|B|<1800$ to aid in feature detection. To study the small-scale features of low magnetic flux in more detail we reduced the dynamic range of the data by one quarter. To partially correct for small variations in magnetic flux due to seeing and for any error in the position of the zero point scale we removed the data points where the magnetic flux was less that $10 \%$ of the maximum value (positive and negative).

\section{Analysis of a small two-ribbon flare}

Active region NOAA 8218 consists of two large sunspots and a number of trailing and surrounding smaller spots. The leading spot is predominantly of negative polarity and was observed by the SVST. Figure 1 shows the region. The upper left panel shows an EIT (Fe XII $\sim 195 \AA$ ) image and the upper right panel shows an MDI continuum image. In both cases the SVST FOV is overlaid. The data maximum of the EIT image has been scaled to $20 \%$ of the original in order to display the larger loop scale structure which is obscured by the very bright flare region. The lower left panel shows an enlargement of the SVST FOV taken in the $\mathrm{H} \alpha$ line center. The overlaid box shows the cutout region used for Figs. 2-4. The lower right panel shows the same cutout enlarged and the overlaid box shows the region selected for the intensity evolution plots of Fig. 5. There

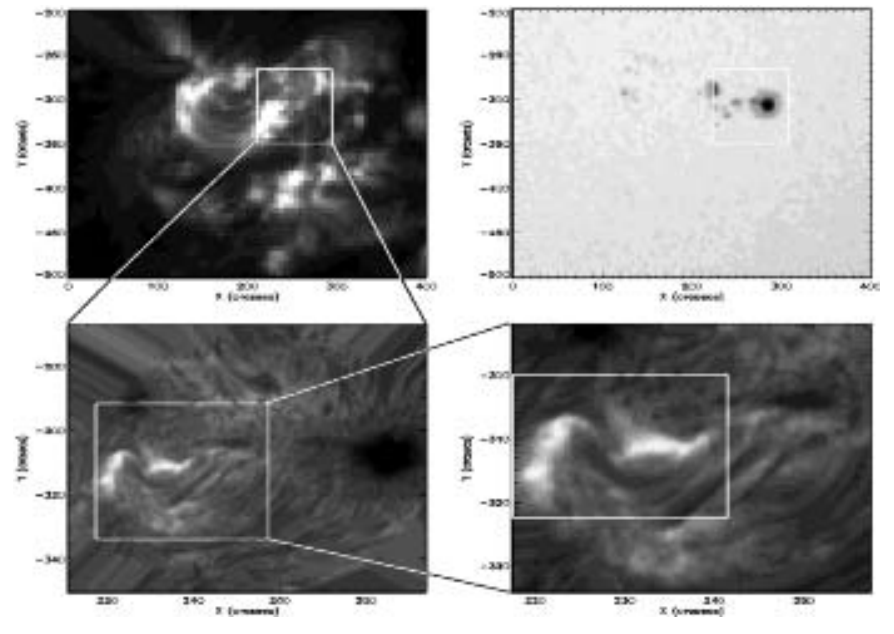

Fig. 1. Identification of analysed regions in NOAA8218. Upper left panel: EIT $195 \AA$ Amage taken at 14:51 UT. The SVST field of view is overlaid Upper right panel: MDI continuum image taken at 14:50:34 UT. The SVST field of view is overlaid. Lower left panel: SVST H $\alpha$ corresponding field of view image taken at 14:57 UT. The overlaid box shows the area of Figs. 3 and 4. Lower right panel: SVST $\mathrm{H} \alpha$ close up of area shown in Fig. 3. The overlaid box shows the area analysed in Fig. 5.
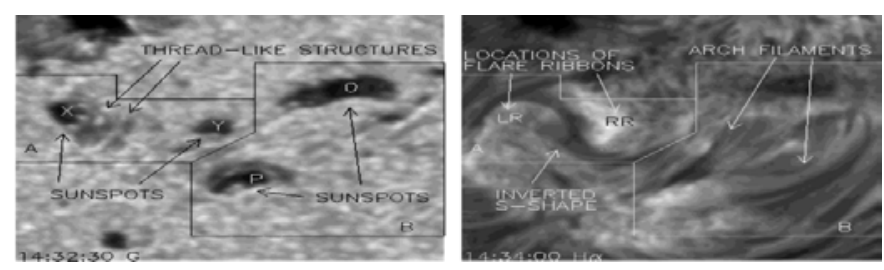

Fig. 2. Figure showing the locations of the main features discussed in the text. The flaring region $\mathrm{A}$, and non-flaring region $\mathrm{B}$, are marked on both images. Left panel: $G$ band image at 14:32:30 UT. The two sunspots are denoted $\mathrm{X}$ and $\mathrm{Y}$. Thread-like structures between them and close to $\mathrm{X}$ are marked. Right panel: $\mathrm{H} \alpha$ image at 14:34:00 UT. The inverted S-shaped feature is pointed out as are several arch filaments in region $\mathrm{B}$. The approximate locations where the flare ribbons brighten are marked with LR and RR.

is considerable obvious activity evident including pore movements, emerging flux, brightenings etc. in the region.

Figure 2 shows a $G$-band and $\mathrm{H} \alpha$ line centre image taken at 14:32:30 UT and 14:34:00 UT, respectively. This figure has been prepared in order to aid identification of the features that are discussed throughout this paper. In the subsequent text, when we refer to, e.g., thread-like structures, we are referring to those identified in this figure. The area shown is a 40.5 by 42.2 arcsec subregion cut out from the full field of view as shown in Fig. 1. Solar North is up and East is to the left. In both images two emerging flux regions (marked A and B) have been outlined. The flare studied in this paper occurred in region A. In the $G$-band image, two sunspots (marked X and Y) are arrowed and in the $\mathrm{H} \alpha$ image the approximate locations of the flare ribbons are arrowed (LR and RR). Below we discuss the relationship between these features and the magnetic field. We also discuss the relationship between the dark thread-like structures (arrowed in the $G$-band image) and the overlying $\mathrm{H} \alpha$ inverted S-shaped structure (arrowed in the $\mathrm{H} \alpha$ image). Some 
arch filaments in region B have also been marked connecting the sunspot $\mathrm{P}$ and the elongated dark sunspot $\mathrm{O}$.

Figure 3 shows the flare development in the $\mathrm{H} \alpha$ line center and $-700 \mathrm{~m} \AA$ blue wing. The area shown is the same as in Fig. 2. The Figs. show the morphology of the dark filaments as they develop through the sequence. At 13:38:04 UT there appear to be two or three arched structures interacting in the image. Two curved structures (labelled numbers 1 and 2 in the image) extend from the North-East towards the image center and a further structure (number 3 ) curves from the center towards the West of the image. It is not immediately clear whether this last structure is separate or whether it is connected to number 1 in a twisted inverted S-shape underneath structure 2. As the sequence progresses this inverted S-shape becomes more pronounced and structure 2 appears to merge with structure 3 or disappear. By 14:48:12 UT only a single inverted S-shape remains clearly visible. As the flare occurs (see the image at 14:52:55 UT) this inverted S-shape seems to separate such that two dark structures appear clearly in the rest of the observations (e.g. labels 1 and 2 in the 14:57:34 UT image). Such a scenario may be evidence for the stressing and shearing of twisted magnetic flux and subsequent separation after magnetic reconnection disrupts the magnetic field in the corona. This is discussed further in Sect. 4. The flare itself is of short duration. Brightenings start to appear around the $\mathrm{H} \alpha$ ribbon locations around 14:43 and are visible in the 14:48:12 UT image. The flare ribbons reach maximum intensity simultaneously at 14:52:55 UT and the main phase is already over by 14:57:34 UT although low level brightenings continue to occur throughout the rest of the observing sequence. In the notation introduced above, LR refers to the location of the left (Eastern) ribbon and RR to the location of the right (Western) ribbon. North West of RR there is an elongated structure $(\mathrm{O})$ which appears connected by $\mathrm{H} \alpha$ filaments to a spot (P) just to the South West of RR. In Sects. 3.1 and 3.2 we discuss the temporal development and interactions of these regions which may provide the key to the production of the flare. The areas of the regions are those marked A and B in Fig. 2 and contours have also been overlaid on the 14:38:53 UT H $\alpha$ image in Fig. 4.

Figure 4 shows the development of the emerging flux region in the $G$-band, magnetogram and $\mathrm{H} \alpha$ line center images before and after the flare. The area shown is the same as Fig. 3. The example images shown cover the period 12:48:26 UT to 16:22:13 UT and were selected for their quality. The images are interspersed with examples of magnetogram contours overlaid on nearly time-coincident $G$-band and $\mathrm{H} \alpha$ images (see the images labelled with $\mathrm{B} / \mathrm{H} \alpha$ and $\mathrm{B} / \mathrm{G}$ ). In these images white indicates positive polarity and black indicates negative polarity. The main contour level is $400 \mathrm{G}$. However, in the flaring region, an additional contour at $220 \mathrm{G}$ has also been plotted to better reveal the lower magnetic flux structures discussed below.

Comparison of the initial and final $G$-band images at 12:49:33 UT and 16:20:13 UT indicates that the region is one of emerging flux. Clearly there is a growth in the number of dark regions confirmed also by an increase in the number of areas of concentrated magnetic flux in the magnetograms at 13:08:16 UT and 16:22:13 UT. From the 14:55:53 UT $G$-band image with overlaid magnetic flux contours it is clear that the two sunspots (noted as $\mathrm{X}$ and $\mathrm{Y}$ in Fig. 2) are associated with magnetic flux of opposite polarities. Note the white positive magnetic flux contours encircling $X$ and the black negative polarity contours encircling Y. From the 14:55:53 UT H $\alpha$ image with overlaid magnetic flux contours it is also clear that the $\mathrm{H} \alpha$ flare ribbons are located on top of and around these opposite polarity sunspots. Note how the flare ribbon (denoted LR above) occurs over and to the South of the white positive polarity contours associated with sunspot $\mathrm{X}$, and the flare ribbon (denoted RR above) occurs over the black negative polarity contours associated with sunspot Y. The same features are visible in the Ca II K-line data (not shown here) prior to the flare but are masked by the brightenings seen at the flare peak time. We examined the spatial locations of the $\mathrm{H} \alpha$ flare ribbons using contour overlays and found that although the flare is more intense in the $\mathrm{H} \alpha$ image than in the $\mathrm{K}$-line image, the $\mathrm{K}$-line brightenings appear well correlated with the $\mathrm{H} \alpha$ ribbons. These findings are consistent with the footpoints being heated by the bombardment of high energy electrons and/or thermal conduction. The structure connecting the footpoints could have pre-existed or been formed during magnetic reconnection.

The 14:55:53 UT $G$-band image with magnetic flux contour overlays also shows that the $\mathrm{H} \alpha$ arch filaments in region $\mathrm{B}$ connecting the elongated dark region, $\mathrm{O}$, and the sunspot, $\mathrm{P}$, are rooted in opposite polarities. Black negative polarity contours encircle $\mathrm{O}$ and white positive polarity contours encircle $\mathrm{P}$.

Note the dark thread-like regions between the two spots and to the right of the spot denoted $\mathrm{X}$ (circled in the 12:49:33 UT $G$-band image). These thread-like structures grow, develop and darken throughout the $G$-band sequence of images and do not appear to be related to the brightenings in $\mathrm{H} \alpha$. The thread-like structures appear nearly perpendicular to the centre-to-centre line connecting the two opposite polarity sunspots. However, by comparing the 13:21 and 14:32 UT $G$-band images, it is possible to see that the orientation of these features changes so that they are pointed further towards the South East i.e. the angle with the centre-to-centre line even increases before the flare. In Sect. 3.2 we demonstrate that these dark structures relate to magnetic flux which is emerging between the two main spots. These structures are of a comparatively lower magnetic flux which can only be seen through reducing the dynamic range of the magnetograms (as discussed above). This may also imply that they are mainly horizontal structures with a lower longitudinal magnetic field. These findings indicate that the emergence of new magnetic flux almost perpendicular to the overlying magnetic field developed a strongly sheared magnetic field configuration, as evidenced by the inverted S-shaped filament, and caused the flare.

Having established that the flare ribbons LR and RR occur on top of and around the sunspots X and Y, we use the labels LR and RR throughout the rest of the paper. From Fig. 4 and from the movies we generated we noted an interesting movement of RR towards LR. This is an unusual motion for an emerging flux region and may be evidence of twisting of the magnetic field. We return to this point in Sect. 3.2. 

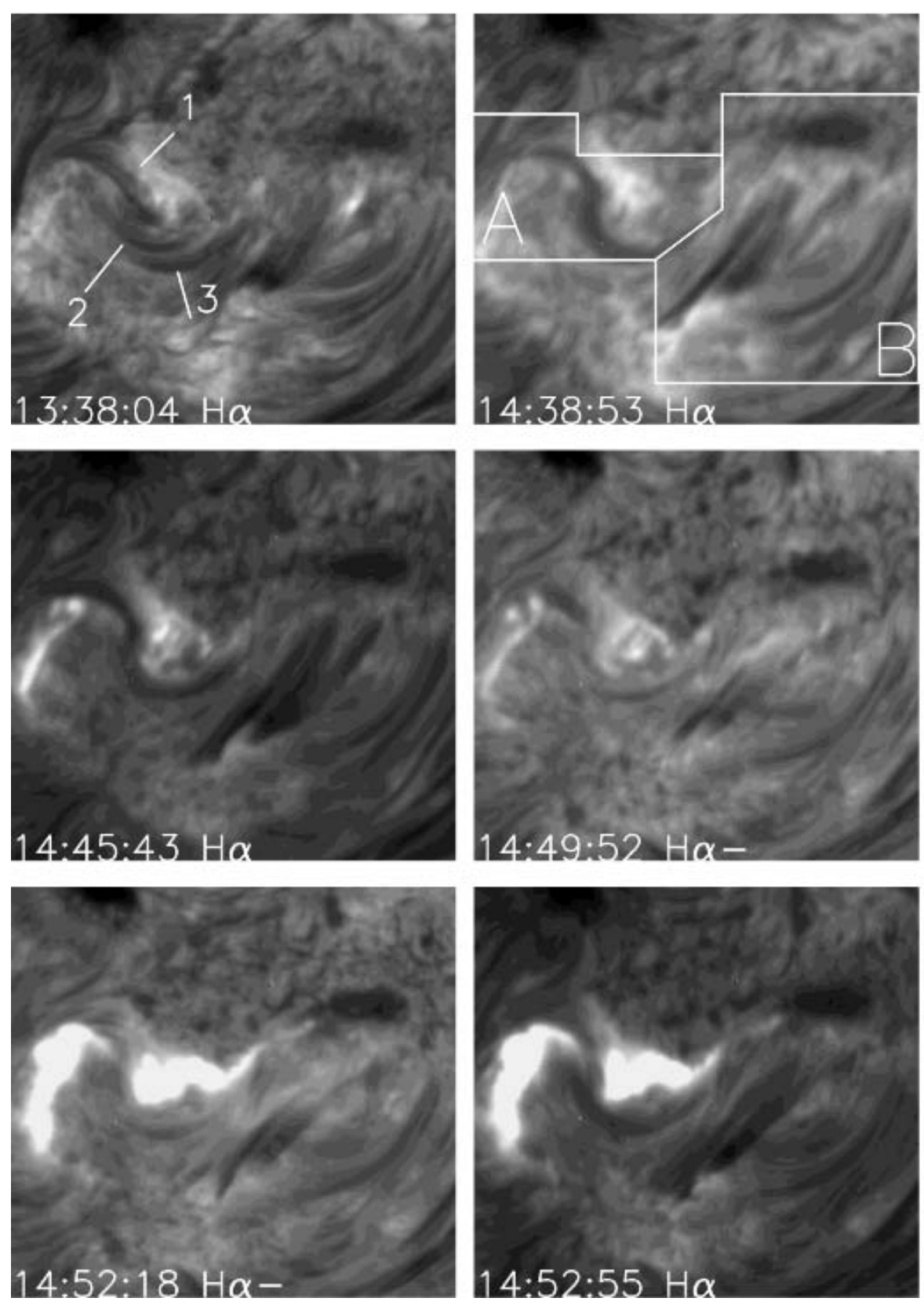

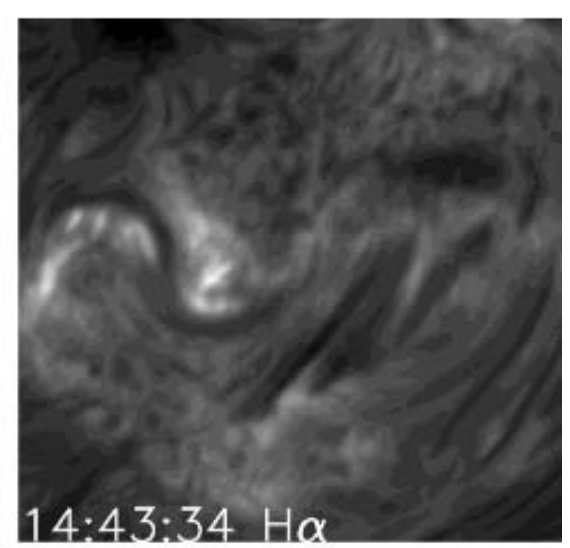

Fig. 3. 40.5" by 42.2" cutout of the observed FOV between 13:38 and 15:02 UT in the $\mathrm{H} \alpha$ line centre and -700 mA blue wing. The blue wing images are denoted by H $\alpha$-and the regions A and B are outlined. On the 13:38:04 UT image the arrows 1, 2 and 3 point out the filament structures before the flare. In the 14:57:34 UT image the arrows 1 and 2 point out the two structures which remain after the flare.

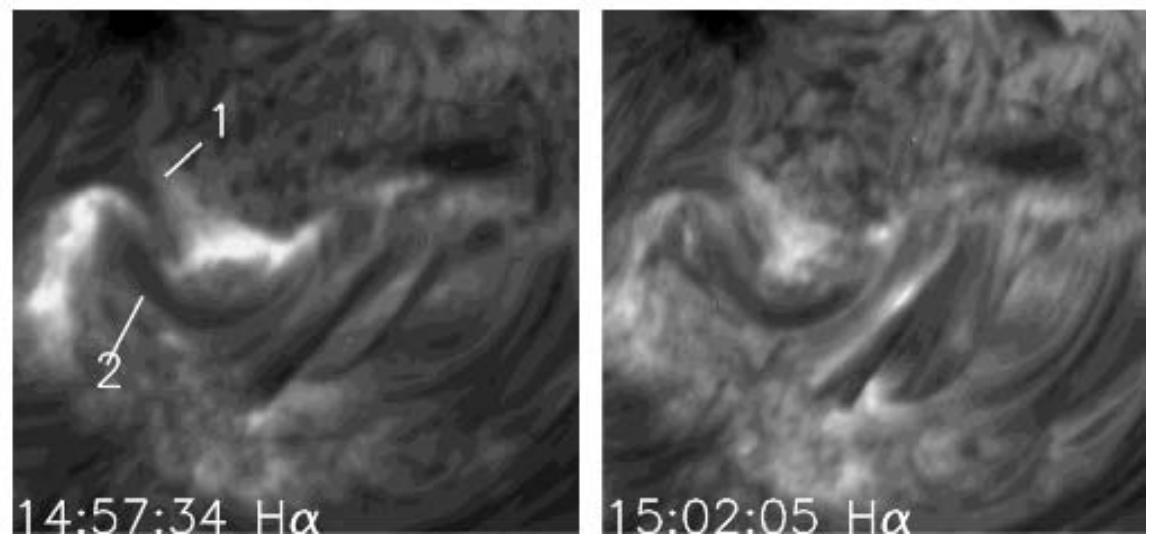



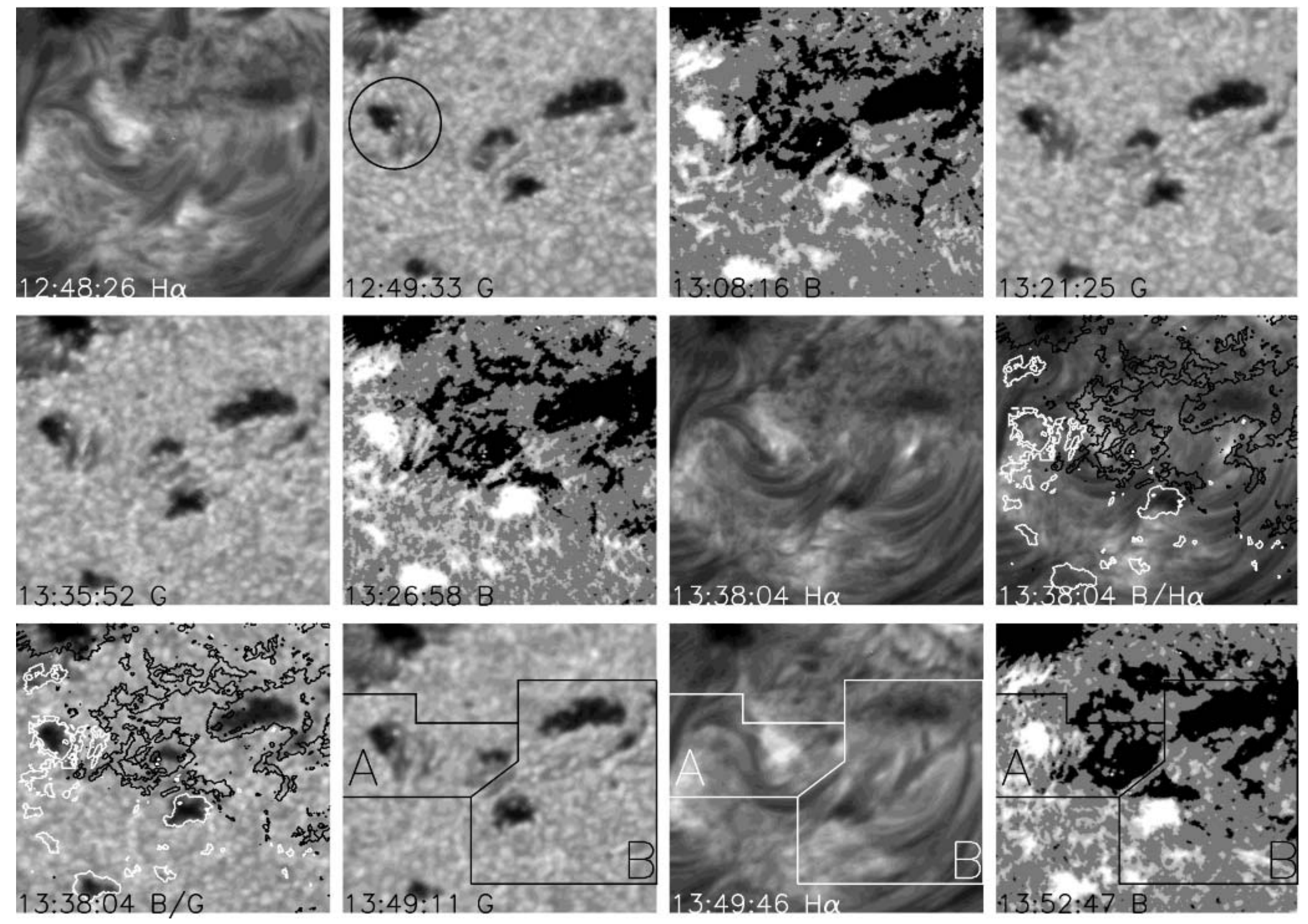

Fig. 4. 40.5" by 42.2" cutout of the observed FOV between 12:48 and 16:22 UT. Magnetograms together with G-band and H $\alpha$ images are shown. Close to time coincident magnetograms are overlaid on some of the images and are labelled $\mathrm{B} / \mathrm{G}$ and $\mathrm{B} / \mathrm{H} \alpha$. The observation times are shown and the regions $\mathrm{A}$ and $\mathrm{B}$ are outlined. 

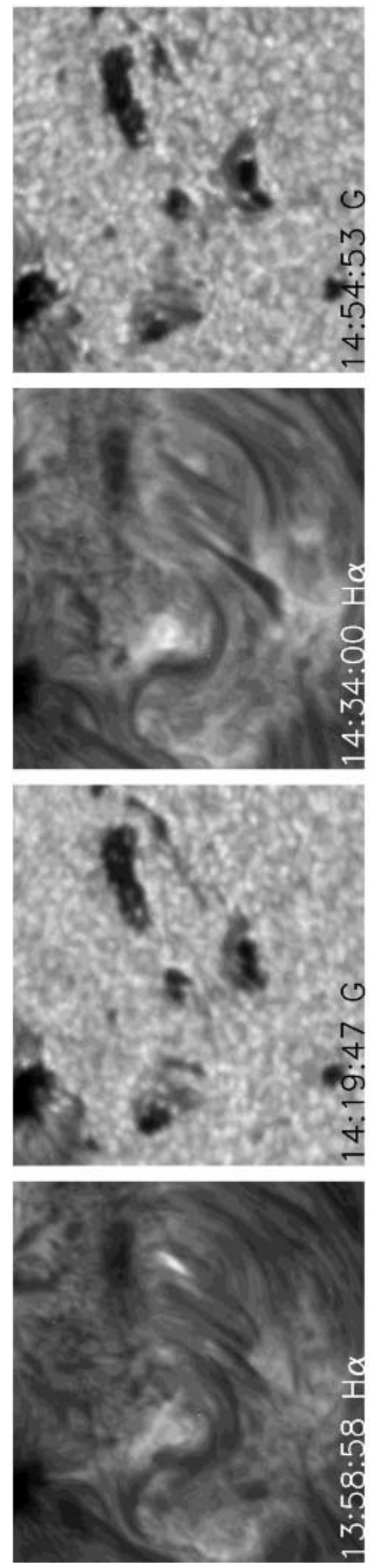
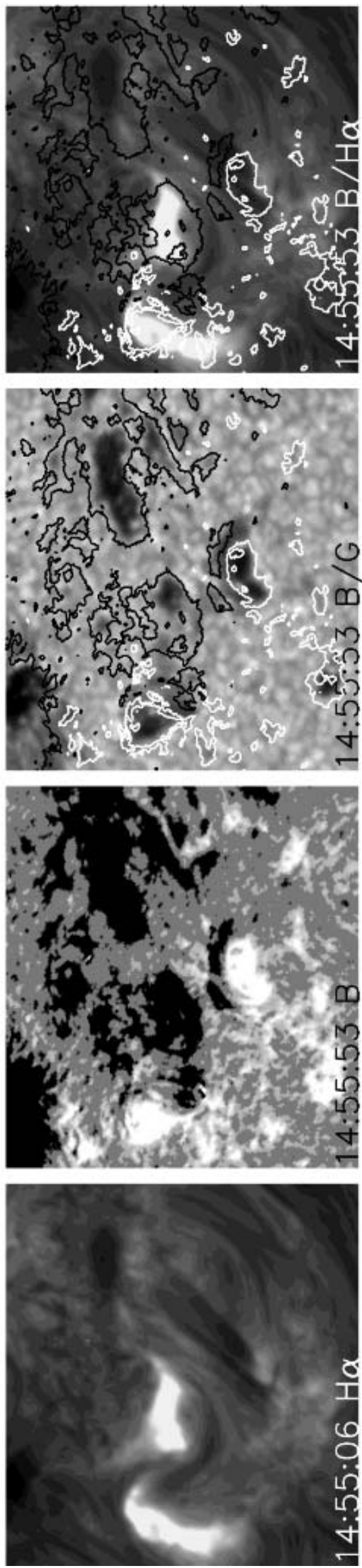
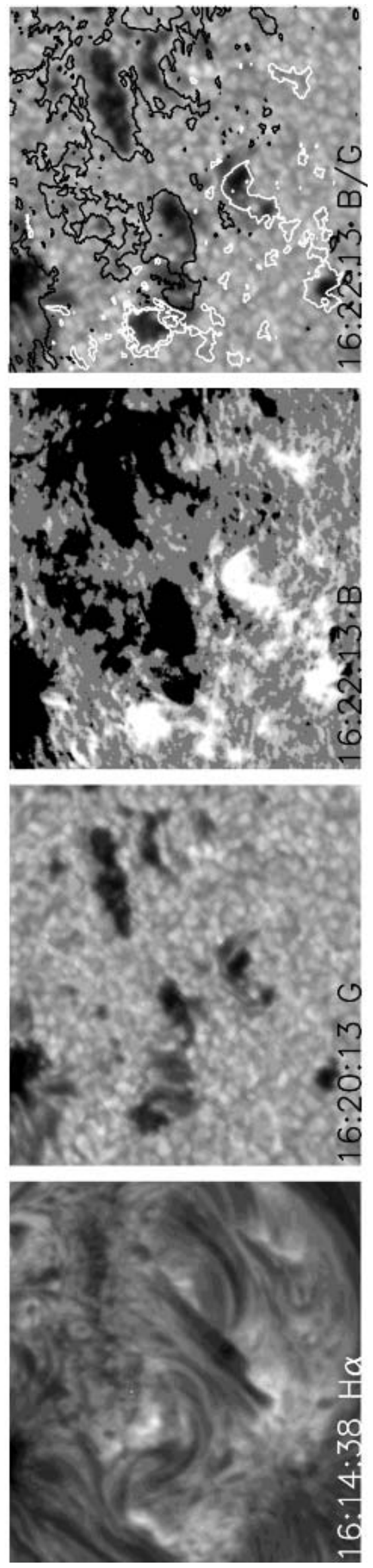

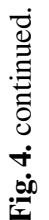




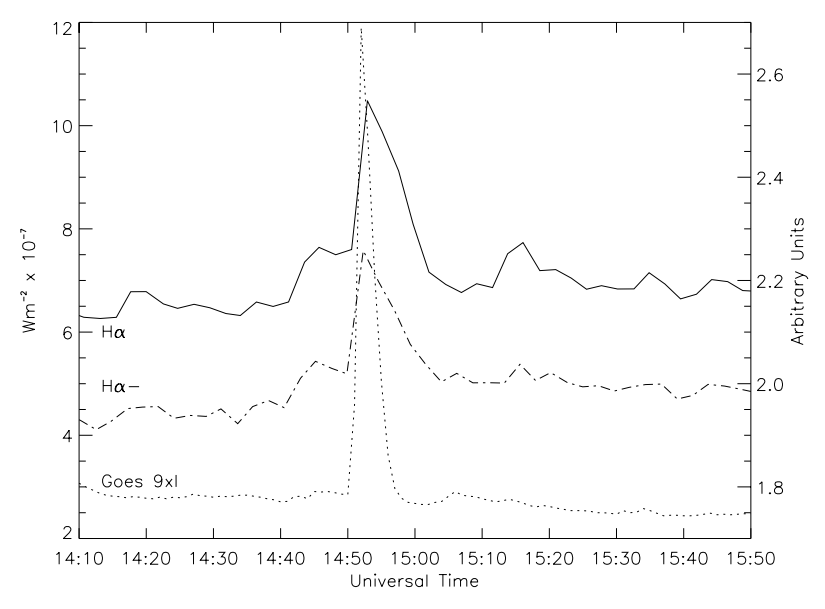

Fig. 5. GOES-9 1 min X-ray flux light curves. The left $y$-axis is in flux units of $\mathrm{Wm}^{-2} \times 10^{-7}$. Light curves for the $\mathrm{H} \alpha$ line center and $-700 \mathrm{~m} \AA$ blue wing are also plotted with the right $y$-axis in arbitrary units. These are marked as $\mathrm{H} \alpha$ and $\mathrm{H} \alpha$-, respectively. They are totals over a region of $\sim 23.7$ by 22.0 arcsec shown by a box overplotted on the 14:52:55 UT image in the lower right hand panel of Fig. 1.

\subsection{Temporal development of the $\mathrm{H} \alpha, X$-ray flux and magnetic field}

Figure 5 shows light curves for the $\mathrm{H} \alpha$ line center and $-700 \mathrm{~m} \AA$ blue wing. The cutout region is a $\sim 23.7$ by 22.0 arcsec subregion shown as a box on the 14:52:55 UT image of Fig. 1 (lower right hand panel). The area was chosen to include the full extent of the $\mathrm{H} \alpha$ flare ribbons. The flux is the total flux within the region, in arbitrary units (right hand $y$-axis). Universal time is given in 10 minute intervals along the $x$-axis from 14:10 UT until 15:50 UT. The curves are labelled as $\mathrm{H} \alpha$ and $\mathrm{H} \alpha-$.

The GOES-9 (Geosynchronous Operational Environmental Satellites) 1 min X-ray flux light curve in the XL band (1$8 \AA$ A) has been overplotted. The flux units are $\mathrm{Wm}^{-2} \times 10^{-7}$ (left $y$-axis). The flare is clearly visible in each curve and as with $\mathrm{H} \alpha$ the GOES X-ray flux peaks at 14:52 UT. The GOES classification for the X-ray flux peak is $\mathrm{C} 1.1$. The X-ray flare duration is approximately $8 \mathrm{~min}$ which is slightly shorter than in $\mathrm{H} \alpha(\sim 20 \mathrm{~min})$.

The main phase of the flare shows a typical impulsive nature in both wavelengths. A precursor brightening can be seen in $\mathrm{H} \alpha$; it starts around 14:42 UT and attains a small peak around 14:46 UT. The GOES flux shows no obvious brightening at this time, but shows fluctuations consistent with the fluctuations in the $\mathrm{H} \alpha$ curve. These precursor brightenings in $\mathrm{H} \alpha$ are identified as small bright points located close to the sheared filament in both the opposite polarity regions. The first brightening occurred at 13:21 UT and many other points successively brightened until 14:50:34 UT and then explosively flashed at the 14:52 UT main peak. Note that we find neither filament motion nor separating motion of the two flare ribbons in this flare, unlike the typical dynamical flare (Kurokawa 1987).

Figures 6 and 7 show the development of the magnetic field in regions $\mathrm{A}$ and $\mathrm{B}$. The average magnetic flux is calculated for each time in the sequence and the results are displayed as the upper left (positive) and lower left (negative) plots in Fig. 6 for the flaring region (A) and Fig. 7 for the non-flaring
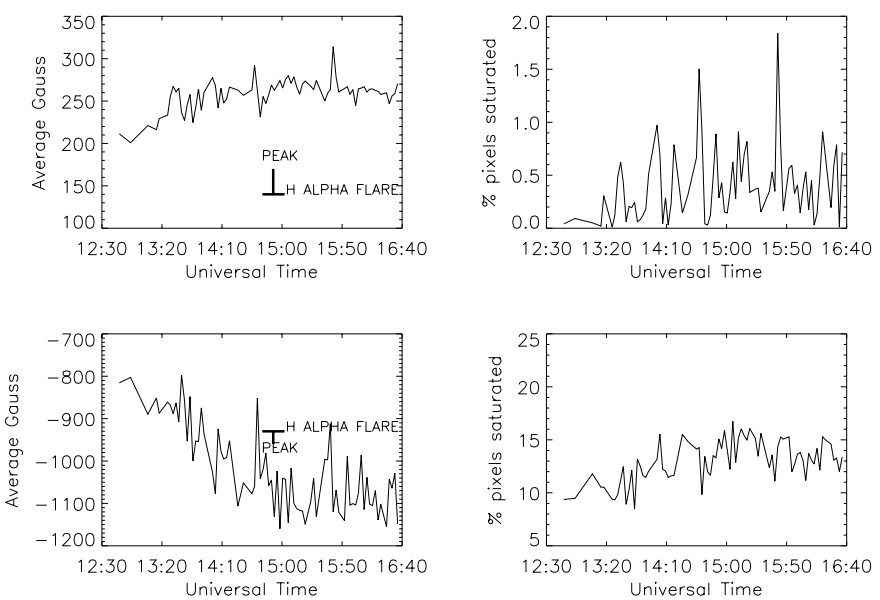

Fig. 6. Time development of average magnetic flux and the percentage of saturated pixels for the flaring region (A in Fig. 4). Upper left average Gauss (+ve). Lower left - average Gauss (-ve). Upper right $\%$ of pixels saturated $(+v e)$. Lower right $-\%$ of pixels saturated $(-v e)$.
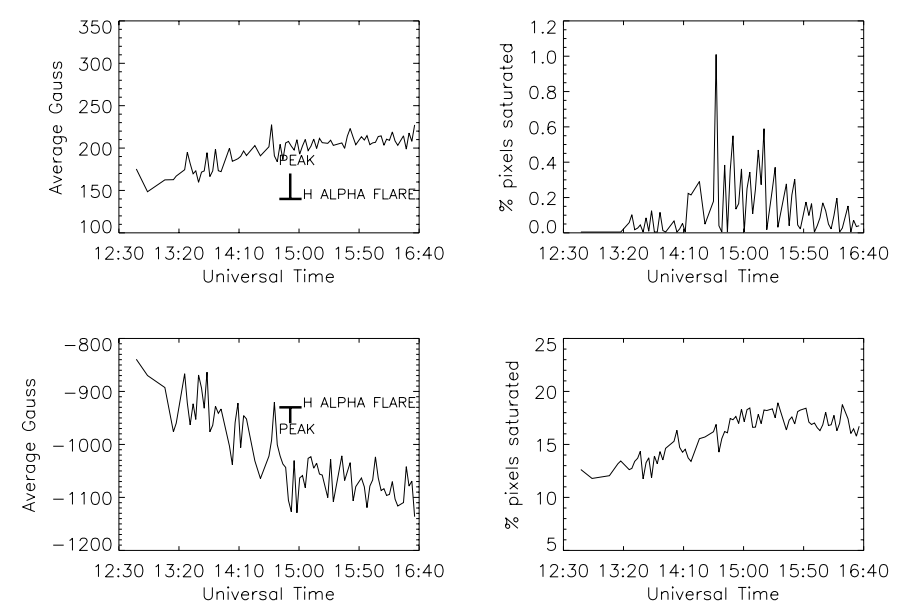

Fig. 7. Time development of average magnetic flux and the percentage of saturated pixels for the non-flaring region (B in Fig. 4). Upper left average Gauss (+ve). Lower left - average Gauss (-ve). Upper right $\%$ of pixels saturated $(+\mathrm{ve})$. Lower right $-\%$ of pixels saturated $(-\mathrm{ve})$.

region (B). The aproximate duration of the $\mathrm{H} \alpha$ flare together with the time of peak emission are also shown. Since the magnetograms do not give us any information on the magnetic field strength above $1800 \mathrm{G}$ this average is a lower limit to the real average. However, we can make an estimate of the accuracy of the average by recording those pixels with values of greater than or equal to $1800 \mathrm{G}$. We call these "saturated pixels" and the $\%$ of the total number of pixels within the area which are saturated is shown for each time in the upper right (positive) and lower right (negative) plots associated with the respective figures.

In the case of the positive polarity flaring region the total \% of pixels which are saturated does not exceed $2.0 \%$. In the case of the negative polarity flaring region the total is substantially larger but does not exceed $17 \%$. In the emerging region to the West the total \% of pixels which are saturated is less than $1.2 \%$ 
for the positive polarity region and less than $20 \%$ for the negative polarity region.

Figure 4 also clearly shows that the positive and negative flux within regions $\mathrm{A}$ and $\mathrm{B}$ are clearer and more intense in the last magnetogram than in the first. Figures 6 and 7 show that the positive polarity region is slightly stronger in the flaring region, $\mathrm{A}$, than in the non-flaring region, $\mathrm{B}$, but that the negative polarity region in the non-flaring region is similar in strength to that in the flaring region. In both A and B the negative polarity region is stronger than the positive polarity region. In addition, the average Gauss of both polarities in both regions increases prior to the flare before apparently levelling off. The positive values increase by a factor of around 1.3 and 1.2 by the time of the flare for the flaring and non-flaring region, respectively. The negative values also increase by a factor of about 1.3 and 1.2 for the two regions by the same time. Also, the percentage of the total number of positive polarity points which saturate increases during this period from 0 to about $1.5 \%$ for region $\mathrm{A}$ and from 0 to about $1.0 \%$ for region $\mathrm{B}$, while the total number of negative polarity points which saturate increases from about 10 to $15 \%$ for the flaring region and from 12 to $17 \%$ for the nonflaring region. Note also that a secondary effect of the increase in pixel saturation is that the factor increases quoted for the average Gauss will be lower limits.

Even though there is some difference in the strength of the positive and negative magnetic flux in the two regions and the rate of increase may be slightly faster in the flaring region, the time development is qualitatively similar. The important point to note is that all three pieces of evidence indicate that both the positive and negative flux is increasing (i.e. magnetic flux is emerging) in both the flaring and non-flaring regions, at least until the flare onset time. This suggests that we must consider other possibilities as to why only one of the regions should produce a flare.

\subsection{Feature tracking and correlation analysis}

In order to track the movement of the various features in the flare region we used three separate techniques; timeslices, small feature tracking and a local correlation tracking method (LCTM).

Figure 8 shows timeslice images, in region A, which demonstrate the movement of the negative polarity spot RR towards the positive polarity spot LR. The lower left image of the figure is taken from the G band data at 12:49:33 UT and the upper left magnetogram was taken at 12:44:28 UT. Both images are 40.5 by 42.2 arcsec cutout areas covering the region shown in Fig. 4. A number of "slice-lines" of varying directions were tested to accurately assess the movement of the spot. The absicca of the coordinate frame of the right hand image shows the pixel position along the slice-line and the ordinate shows the time. The chosen slice-line is overplotted on the two left hand images and is the same for both the $G$ band data and the magnetograms. The positive polarity spot therefore sits towards the left hand end of the slice-line near pixel 0 in the slice-image. The negative polarity spot is located close to the right hand side of the slice-line which corresponds to pixel 148 in the
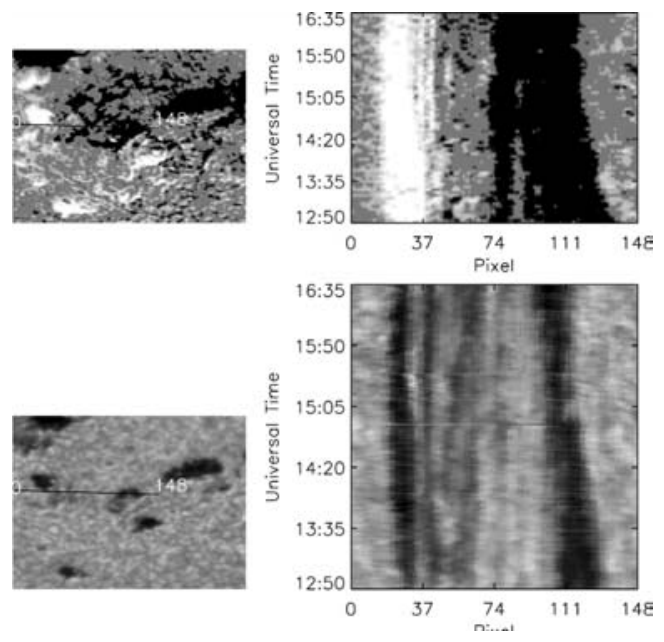

Fig. 8. Timeslice image showing the movement of RR towards LR (see text). The image on the lower left of the figure is taken from the $G$-band data at 12:49:33 UT and the magnetogram in the upper left was taken at 12:44:28 UT. Both are 42.2 by $42.2 \mathrm{arcsec}$ in size. The slice-line is overlaid and the starting pixel (0) and ending pixel (148) are also shown. The absicca of the coordinate frame of the right hand plot shows the same pixel position along the slice-line. The ordinate shows the time.

slice-image. The slice-image clearly shows that the negative polarity region moves along the line so that the leading edge has moved from around pixel 107 to around pixel 93. The trailing edge of the positive polarity spot also moves a little along the slice-line from around pixel 32.5 to around pixel 29.5. Assuming that 1 arcsec represents approx. $725 \mathrm{~km}$ on the Sun's surface this relative distance of 11 pixels corresponds to $\sim 794$ $\mathrm{km}$ and hence a relative velocity of $\sim 59 \mathrm{~m} \mathrm{~s}^{-1}$. This result indicates that if the two spots are magnetically connected, then some shearing of the magnetic neutral line must occur. Note also the stability of the positive polarity spot (the darkest line to the left of the slice image).

Figure 9 contrasts the development of the non-flaring region, $\mathrm{B}$, with that of the flare region A. The $G$-band image and magnetogram are the same as before. Again a number of slicelines were used to accurately assess the direction of movement. Pixel 0 on the slice-line is positioned just ahead of the direction of motion of the positive polarity region, P, and pixel 179 is ahead of the direction of movement of the negative polarity region, $\mathrm{O}$. The dark regions in the $G$ band image show indications of a separating motion which appears more obvious in the timeslice of the magnetogram. The negative polarity feature associated with the upper spot (around pixel 134) clearly moves while the movement of the positive polarity region (around pixel 44) is less obvious. However, even a slightly curved separating path, similar to the shape of the path in the $G$ band timeslice, can be discerned. A bipolar sunspot is also seen moving between the two main spots.

Consider now the darkening of the area between the spots in Fig. 8 (around pixels 37 to 56). We investigated this region in further detail and two further timeslices are shown in Figs. 10 and 11. This time the $G$ band image was taken at 13:35:52 UT and the magnetogram at 13:36:20 UT. These times were chosen 

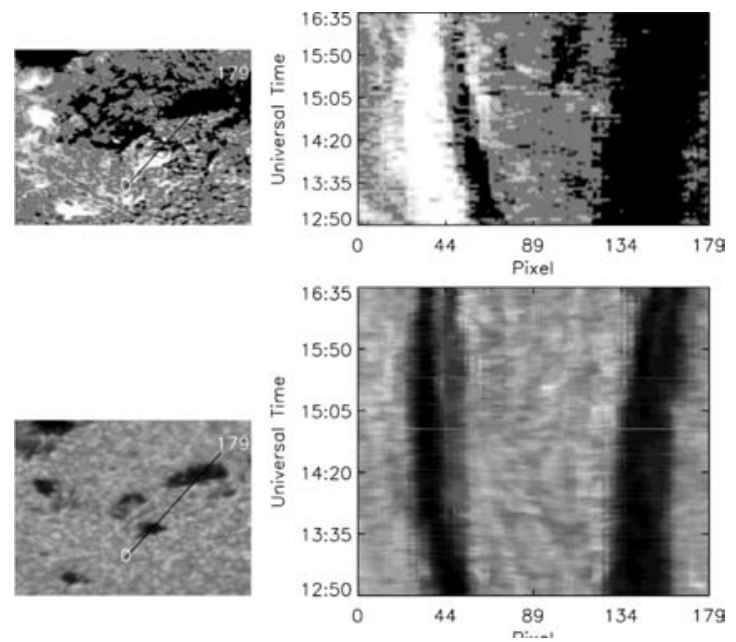

Fig. 9. Timeslice image showing the separation of the structures in the non-flaring region, B. The $G$-band image and magnetogram are the same as before. The slice-line is overlaid and the starting pixel (0) and ending pixel (179) are also shown. The absicca of the coordinate frame of the right hand plot shows the same pixel position along the slice-line. The ordinate shows the time.

in order to examine the relationship between the magnetic field and the dark regions. These regions correspond to the threadlike structures discussed earlier which are visible between the two spots and are emerging almost perpendicular to the line connecting the opposite polarity sunspots. By 13:30 considerable darkening between the spots had already occurred and the thread-like structures are quite well defined (compare this image with the one in Fig. 8, for example). Of course, this darkening is itself clear evidence of emerging flux.

In Fig. 10, we examined the right hand thread. The upper end of the slice-line in this figure corresponds to pixel 0 and the lower end corresponds to pixel 112. By comparison of these timeslice images and the contour overlays in Fig. 4 we determined that this thread is associated with predominantly positive magnetic flux but with a negative polarity region at its lower end. These positive polarity regions appear to separate when observed in the movies and this is reflected in the magnetic field time slice. These observations are consistent with the general picture of emerging flux: when a magnetic flux rope has emerged above the photosphere, the expansion of the structure naturally causes the magnetic footpoints to separate. However, while the right hand dark structure (around pixel 70) appears to be associated directly with negative magnetic flux the positive flux appears to be at the boundary of the thread and the surrounding region (compare the position of the left edge of the dark region in the $G$ band timeslice with the positive separating flux in the magnetic field time slice).

In Fig. 11, we examined the left hand thread. The upper end of the slice-line in this figure corresponds to pixel 0 and the lower end corresponds to pixel 84. Again by comparison of these timeslices and the contour overlays of Fig. 4 we determined that this thread is associated almost exclusively with positive magnetic flux. The dark negative flux that appears in the timeslice around pixel 63 at around 14:10 UT seems to correspond to separate flux moving onto the slice-line from the
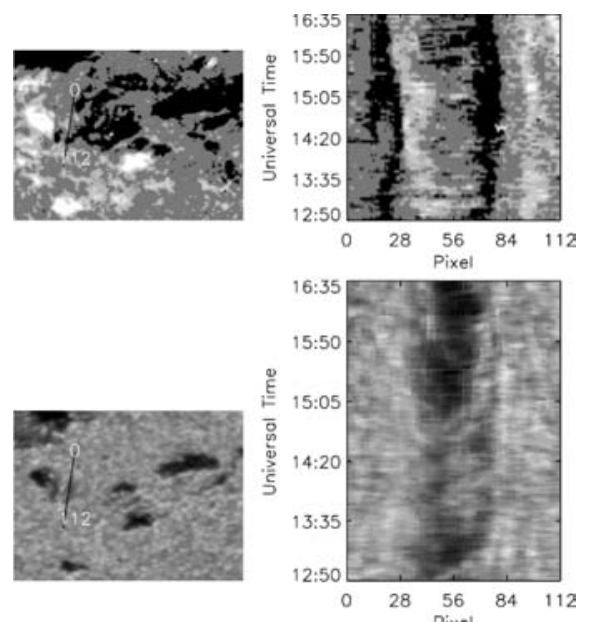

Fig. 10. Timeslice image showing emerging flux between LR and RR. The $G$-band image in this figure was taken at 13:35:52 UT and the magnetogram was taken at 13:36:20 UT. The slice-line is overlaid and the starting pixel (0) and ending pixel (112) are also shown. The absicca of the coordinate frame of the right hand plot shows the same pixel position along the slice-line. The ordinate shows the time.
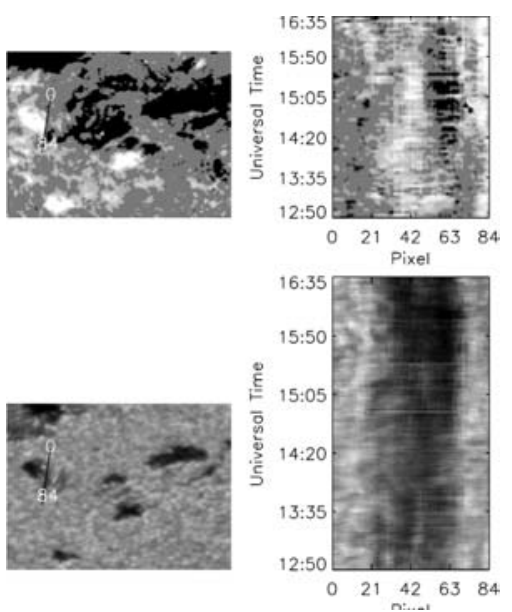

Fig. 11. Timeslice image showing emerging flux between LR and RR. The $G$-band image in this figure was taken at 13:35:52 UT and the magnetogram was taken at 13:36:20 UT. The slice-line is overlaid and the starting pixel (0) and ending pixel (84) are also shown. The absicca of the coordinate frame of the right hand plot shows the same pixel position along the slice-line. The ordinate shows the time.

West. This feature also appears to be curving around the main positive spot. In fact although both thread structures appear to initially emerge almost perpendicular to the line connecting the main spots, the angle between the threads and this line appears to increase before the flare as discussed in Sect. 3. The difficulty in relating the $G$ band and magnetic features is mainly due to the variability in seeing conditions and low field strength in this region. However, these results suggest that this emerging flux developed a sheared magnetic field configuration with the overlying field, as indicated by the inverted S-shaped $\mathrm{H} \alpha$ filament, and caused the flare.

Figure 12 shows a time-slice with a similar orientation to Fig. 8 but using SOHO/MDI full disk data. Although the data is of lower resolution it enables us to both cover the SVST 

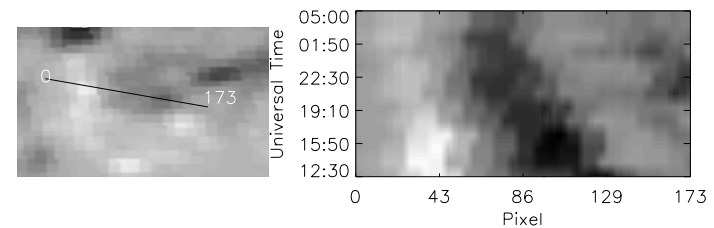

Fig. 12. Timeslice image showing the movement of the positive and negative polarity spots from SOHO/MDI data. The timeslice covers the SVST observations period and extends much later until around 05:00 UT on May 14th.

observations period and investigate the development of the region after $17 \mathrm{UT}$. In this figure the time-slice is extended to 05 UT on May 14. It clearly shows the approach of the negative polarity region to the positive polarity region. Note also that it continues to approach even after the flare has occurred. Later the two spots appear to maintain their separation and analysis of further data up to 17 UT shows that the negative polarity spot also seems to change direction around 5 or 6 UT and eventually positioned itself to the South of the positive polarity spot. Finally the two spots disappear from MDI white light images around $06 \mathrm{UT}$ on the 15 th. This information is important when considering the discussion of Sect. 4.

Having established the general motion of the large regions we applied feature tracking and correlation analysis to the dataset.

The LCTM was applied to an area of $81^{\prime \prime}$ by $84.6^{\prime \prime}$ around the region of interest. The method adopted derives the velocity structure from the dataset by tracking correlations between image sub-areas within a pre-defined box size, search area and temporal distance. These parameters are determined by reducing the difference between results obtained from an even-odd test. That is, results are obtained for a dataset of odd numbered images and also for a dataset of even numbered images. The differences between the results are compared and the parameters which best reduce this difference are selected. For our dataset this procedure produced a box size of 10 by 10 pixels (3.38" by $3.38^{\prime \prime}$ ), a temporal distance of $120 \mathrm{~s}$ on average and a search area of 5 pixels. This last parameter is large enough to trace features with velocities of $9.8 \mathrm{~km} \mathrm{~s}^{-1}$ and is also enough to track motions due to seeing. It is also sufficiently small to exclude correlations with distant boxes.

The results are combined in Fig. 13 which is a composite of several images. The main plot and scale is the velocity map. The numbers on the $x / y$-axes are simply labels separated by the box width/height to give a reference position when referring to the various features. Since the box width is 10 pixels the 240 by 250 pixel image is divided into 24 by 25 boxes. The arrows shows the direction of motion of each box and their magnitudes are proportional to the distance travelled by the box although the absolute value is arbitrary. The underlying image is the divergence image. White areas are regions of positive divergence and dark areas are regions of negative divergence i.e. convergence. The brighter/darker the region the greater the divergence/convergence. For example, the maximum divergence is found around position $(4,3)$. Contours of the $G$ band dark spots are overplotted with levels of $0.195 \%$ and $0.390 \%$.

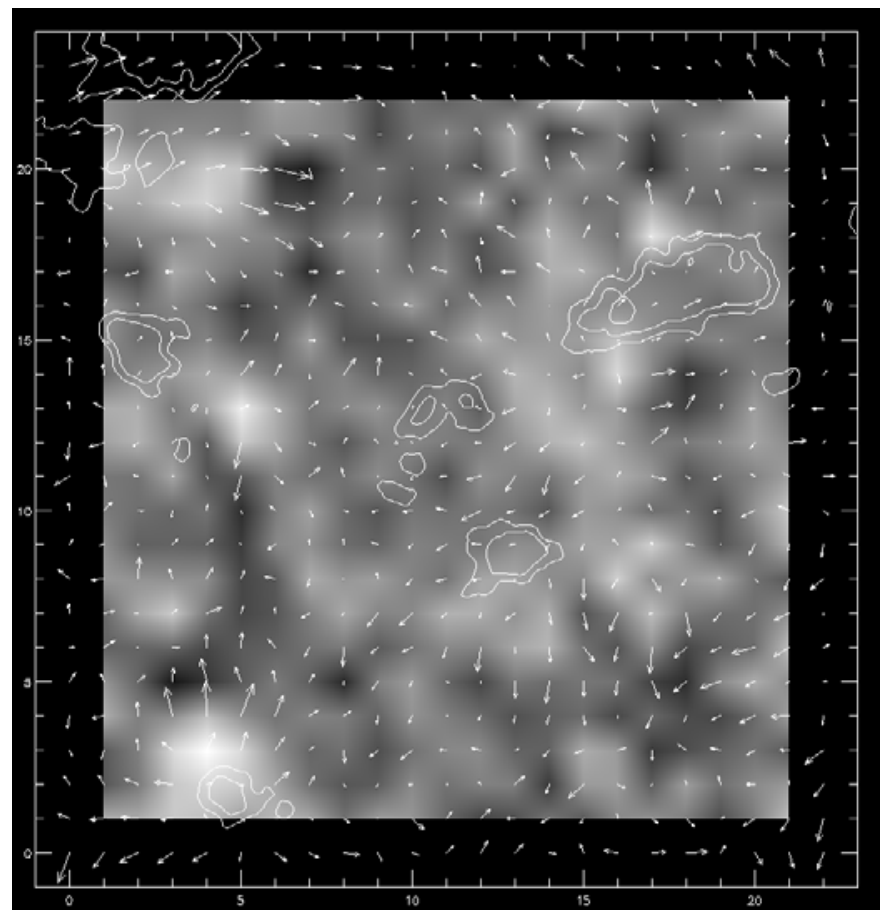

Fig. 13. Results from the local correlation tracking method. The size of the area is the same as Figs. 3 and 4. The velocity map is plotted and the magnitude of the arrows are proportional to the distance travelled by the corresponding box although the absolute value is arbitrary (see text). The arrowhead gives the direction of motion. The underlying image image is the divergence map. White areas are regions of positive divergence and dark areas are regions of convergence. Contours of the $G$ band dark spots are also overplotted with levels of $0.195 \%$ and $0.390 \%$.

The results confirm and extend the findings from the timeslice method. The maximum, average and minimum velocities over the whole observing period (3h46m57s) are 1.679, 0.4729 and $0.02884 \mathrm{~km} \mathrm{~s}^{-1}$, respectively. There is a clear divergence in the region where flux is emerging between LR and RR supported also by strong velocity flows. Since the box size is small it is effective at tracking this motion. RR also shows large velocity arrows at its trailing edge and dark convergence regions between it and LR. Along the line chosen for the time slice analysis of Fig. 7 their is initial convergence before it is overwhelmed by divergence from the region emerging parallel to the neutral line. This is consistent with the timeslice results as RR does not approach this close to LR throughout the sequence. The LCTM method also reveals the separating motion of the two $G$ band dark spots $\mathrm{O}$ and $\mathrm{P}$ in the non-flaring region. As mentioned these regions are connected by dark loop structures in the $\mathrm{H} \alpha$ data. There is also a constant stream of short-lived bright points in both the $G$ band and K-line datasets (not presented here) in this area which seem to move between $\mathrm{RR}$ and spot $\mathrm{P}$ and also around the latter spot and almost parallel to the lower edge of the elongated dark structure, O. More precisely, the flows are not obviously perpendicular to the neutral line between these features as one might expect from an emerging structure. The flows may also have some influence on the movement of RR towards LR. 
Note that Fig. 13 shows almost no velocity motion at the flare site between the positions of the $\mathrm{H} \alpha$ flare ribbons and that the divergence image shows no strong convergent or divergent flows.

Feature tracking was also applied interactively selecting regions in the images and visually following their motion through the dataset. This was found to be very effective for following the motion of the larger spots. In the case of smaller features, such as $G$-band bright points, the technique was of limited effectiveness in the area of interest around the flare site due to the lack of motion in this region and the short duration of the brightenings. However, it was possible to identify and track longer duration brightenings and movements such as the flow pattern between RR and the spot P evident in the LCTM results discussed above. The feature tracking results were substantially the same as the those of the timeslice and LCTM procedures and so have been omitted for brevity.

\subsection{Space based observations}

Figure 14 shows a close-up image of the flare region at the time of peak intensity (14:50:57 UT) obtained by EIT (Fe XII $\sim 195 \AA$ A). The image corresponds to the SVST FOV as shown in Fig. 1 and is 84 by 84 arcsec. An MDI magnetogram has been overlaid on the close-up to aid in cross-identification of features with the SVST dataset. The magnetogram was taken at 14:58:05 UT. Positive polarity regions are denoted by dashed contours and negative polarity regions are denoted by solid contours. Figure 15 shows an SVST H $\alpha$ line center image taken just after the flare peak at 14:57:34 UT (closest in time to the EIT and MDI images). The EIT image has been overlaid as solid contours to show the locations of the brightenings. The contour surrounding LR is about $50 \%$ of the peak intensity of RR. A Yohkoh-SXT image taken at 14:56:06 UT is also overlaid as dashed contours. The percentages for the contour levels are the same in both overlays i.e. $20 \%, 50 \%, 80 \%$ and $100 \%$ of the peak intensity of the image. The images have been processed using EIT and MDI procedures together with D. Zarro's IDL mapping software, both available in SolarSoft.

The MDI contours are in arbitrary units at intervals of 100 between 100 and 1100. Positive polarity regions are denoted by dashed contours and negative polarity regions are denoted by solid contours.

The EIT image shows the flare site in the ultraviolet wavelength range at plasma temperatures of around 1.4 MK. Interestingly the peak intensity appears to lie close to the negative polarity (RR) spot in the MDI images and this is not an effect of the time difference between images as it retains the same location in later MDI magnetograms. Recall that Fig. 1 image also shows extensive larger bright features which can be identified as portions of coronal loops connecting from the predominantly negative region to a largely positive region outside the SVST field of view but visible with EIT/MDI. For example, consider the low level contour below the flare site in Fig. 15. Figure 1 shows that it is clearly identified as the lower part of a larger loop structure(s).

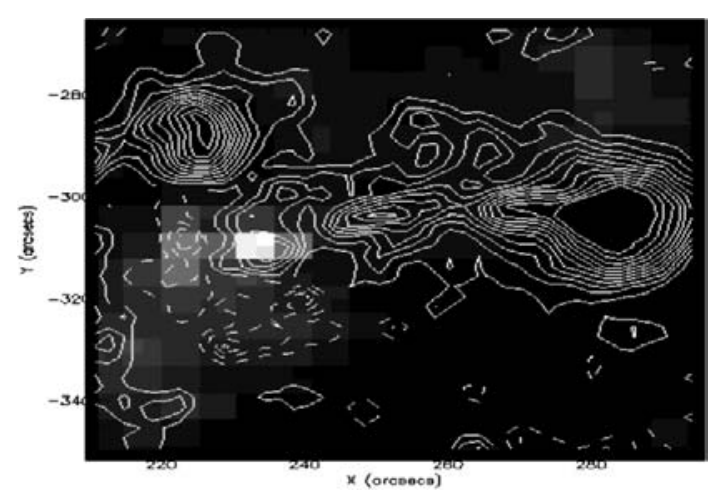

Fig. 14. EIT $195 \AA$ image of NOAA 8218 at the time of flare peak intensity (14:50:57 UT). corresponding to the SVST field of view as shown by the box on the EIT image in the upper left hand panel of Fig. 1. An MDI magnetogram has been overlaid to aid in cross identification of features with the SVST dataset. The magnetogram was taken at 14:58:05 UT. Positive polarity regions are denoted by dashed contours and negative polarity regions are denoted by solid contours.

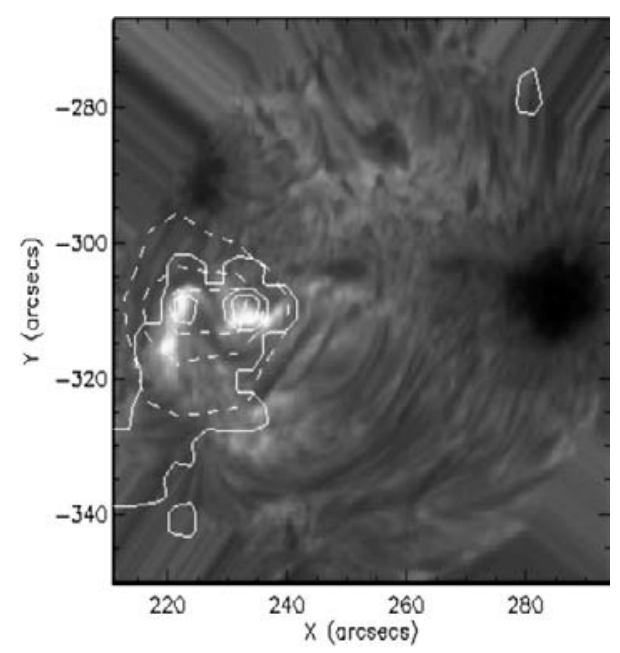

Fig. 15. SVST $\mathrm{H} \alpha$ line center image taken just after the flare peak at 14:57:34 UT (closest in time to the EIT and MDI images of the previous figure). The EIT image has been overlaid as solid contours to show the locations of the brightenings. The contour surrounding LR is about $50 \%$ of the peak intensity of RR. A Yohkoh-SXT image taken at 14:56:06 UT is also overlaid as dashed contours. The percentages for the contour levels are the same in both overlays i.e. $20 \%, 50 \%, 80 \%$ and $100 \%$.

The precise locations of the foot points of these larger loops are not easily established as the EIT spatial resolution is not high enough for a detailed comparison with the SVST data. However, the coronal loop seems to cross above the emerging flux region at an angle perpendicular to the neutral line. Recall that magnetic flux is also emerging in this direction between the flare ribbons. This then might suggest that the flare is a product of interacting loops in a magnetic quadrupole configuration (see e.g. Aschwanden et al. 1999 and references therein) or, alternatively, in a similar scenario to that proposed for homologous flares by Ranns et al. (2000) i.e. an emerging loop/overlying loop interaction.

We also derived the emission measure for the soft X-ray brightening using procedures developed for Yohkoh and 
available in SolarSoft under the SXT branch. The SXT image was taken with the AlMg filter. However, unfortunately we could not use the filter ratio method of Hara et al. (1992) as the closest image in time taken by a different filter was around $20 \mathrm{~min}$ before the flare onset. Therefore we resorted to assuming an average temperature for the post flare X-ray loops of around $10 \mathrm{MK}$. This value is representative for post flare loops according to Kamio et al. (2003). The region of the flare was defined as the area where the intensity is greater than $10 \%$ of the peak intensity i.e. $1428 \mathrm{DN} \mathrm{s}^{-1}$ pixel $^{-1}$. Under this criterion we find an area of $\sim 4.73 \times 10^{18} \mathrm{~cm}^{2}$. From the total intensity of this region we derived a volume emission measure of $\sim \log E M=47.75 \mathrm{~cm}^{-3}$. If we assume that the flare brightening depth is proportional to the square root of the surface area then the volume is $1.03 \times 28 \mathrm{~cm}^{3}$ and hence the density is $7.4 \times 10^{9} \mathrm{~cm}^{-3}$. Of course if the filling factor is less than unity then the density will be correspondingly higher.

Assuming this density we can make a simple estimate of the thermal energy content via $E_{\text {th }}=\left(3 k_{\mathrm{B}} T_{\mathrm{e}} E M\right) / N_{\mathrm{e}}$, where $N_{\mathrm{e}}$ is the electron density, EM is the volume emission measure, $T_{\mathrm{e}}$ is the electron temperature and $k_{\mathrm{B}}$ is Boltzmann's constant. We derive a value of $3.15 \times 10^{29} \mathrm{ergs}$. The magnetic energy can then be estimated as $2.1 \times 10^{29} \mathrm{ergs}$ from the relation $E_{\mathrm{mag}}=\frac{2}{3} E_{\mathrm{th}}$ (see e.g. Shibata \& Yokoyama 2002).

\section{Discussion and model}

First let us summarise our observational findings.

- The $\mathrm{H} \alpha$ flare ribbons occur at opposite magnetic polarity footpoints.

- Thread like sunspot features, which mainly have a horizontal magnetic field, appeared in a direction nearly perpendicular to the line connecting the bipolar sunspots. This implies that new magnetic flux emerged perpendicularly to the overlying preexisting magnetic field and thus developed a strongly sheared magnetic field configuration which triggered the flare occurrence. This is very similar to cases found before for much stronger flares (Kurokawa 1987).

- The negative polarity footpoint (RR) moves towards the positive polarity footpoint (LR) at a slight angle from the center to center line. This is confirmed by both correlation analysis and feature tracking.

- An inverted S-shaped $\mathrm{H} \alpha$ dark filament was formed in the flare region shortly or about $0.5 \sim 1$ hours before the flare. This indicates the development of a strongly sheared magnetic field along the polarity boundary line.

- Emerging flux is also identified in this direction in the $G$-band and Fe I images.

- Magnetic field increases (of both polarities) were clearly identified before the flare.

- The highest intensity flare brightening in EIT is observed above the footpoint of greater magnetic field strength.

- EIT images indicate higher temperature connectivity out of the SVST field of view. However, this is principally in the form of larger loops connecting to the positive polarity companion sunspot.

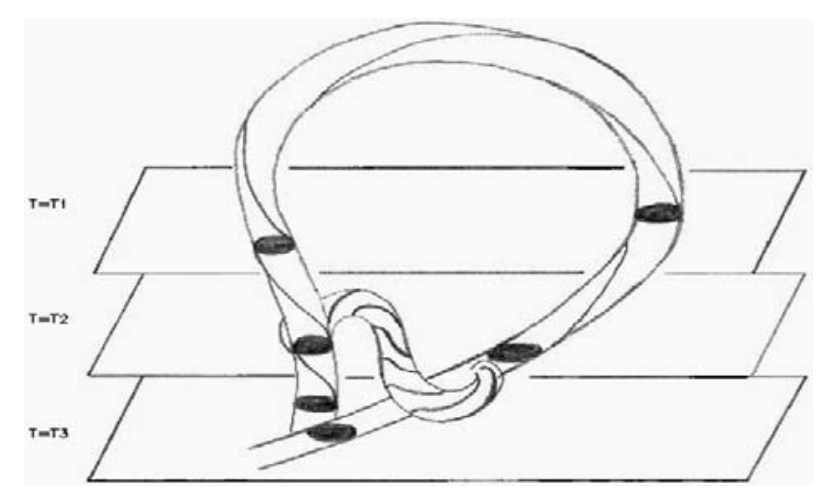

Fig. 16. Schematic drawing of emerging twisted magnetic flux tube as discussed in the text.

- These larger coronal loops appear to cross above the opposite polarity pair approximately parallel to the neutral line.

Any model based on reconnection or untwisting of the magnetic field must be consistent with the essential features we observed.

Figure 16 shows a schematic model of the emergence of twisted magnetic flux which we propose to account for these observations. The figure shows a twisted magnetic flux tube and connecting "branch" emerging above the photosphere. The box cut through the structure indicates the position of the photosphere at two different times in the observing sequence. As the flux tube emerges its footpoints will initially separate. However, the schematic model is designed such that the tube above LR is vertical and the tube above RR is twisted to an half $\Omega$ shape. Hence, at a later time, LR is still located at the same position but RR has an apparent motion towards LR. Since the angle of approach is not quite center to center the structure must twist (at least initially) to the south of LR. To establish the subsequent motion of RR we checked MDI full disk continuum and magnetogram data (some of which is shown in Fig. 12) and found that RR continued to approach LR several hours after the SVST observations were completed eventually stabilising in position near LR before they both disappear. This is the reason our main flux tube takes the shallower line and direction in the figure.

A connecting "branch" is also shown twisting in an inverted S-shape from one trunk of the flux tube in an upward and then downward direction to attach to the other trunk. Initially none of this "branch" is observed but at a later time dark structures are seen emerging parallel to the neutral line and close to LR. As the structures emerge the footpoints separate (as observed in the time slice Fig. 10). The magnetic field strength is lower in this region so the field lines close to the strong positive polarity branch may obscure any weak magnetic flux associated with the emerging branch which does not show any separating motion (Fig. 11). Alternatively, the weak longitudinal field may suggest that the branch mainly has a horizontal magnetic component. At greater heights in the atmosphere $\mathrm{H} \alpha$ dark filaments are located along the neutral line close to the field lines of the main twisted structure. The emergence of this branch almost perpendicular to the overlying bipolar field along the main flux rope, causes a quick development of a sheared magnetic field 
configuration which is observed as the inverted S-shaped structure in $\mathrm{H} \alpha$.

We do not claim that this simple model is a unique interpretation of the data. However, it does qualitatively explain all of the observed features and accounts for them with one connected magnetic structure. Another possibility is that the emerging flux tube is connected below the photosphere to the non-flaring emerging flux region passing through both footpoints in an arch i.e. both regions are part of one emerging structure. This single structure could itself be a loose branch from a much larger emerging trunk at the location of the large sunspot in the field of view (similar to the schematic models proposed from observational studies of flare productive regions by Ishii et al. 1998 and Ishii et al. 2000). Alternatively, the structure may just be composed of magnetic threads penetrating the surface at different locations as proposed from numerical computations of magneto-convection by Stein and coworkers (see e.g. Stein \& Nordlund 2002). In these cases the footpoint separation of the non-flaring region is explained by the emergence of the connecting loop or thread.

The logical next question to ask is how such a magnetic configuration could result in the occurrence of the flare? A number of scenarios could be imagined but here we mainly consider the possibility of reconnection within the rising twisted flux rope itself or magnetic reconnection between the twisted magnetic field and the overlying coronal field.

Note that the $\mathrm{H} \alpha$ two-ribbon flare did not show the usual separation of footpoints that is expected from an emerging flux rope. Also, we were unable to detect any obvious flare trigger, for example filament ejection, and these observations imply that the flare occurred in a confined loop system. This suggests that reconnection within the twisted flux rope due to the kink instability could be the cause of the flare. In this case we might expect to observe further evidence of some untwisting of the magnetic field such as a change in the geometry of plasma material situated on the magnetic loop. In fact we do observe a contact and separation of the S-shaped $\mathrm{H} \alpha$ filament at the time of the flare. However, it appears to return to the pre-flare geometry after the event so if it is a signature of untwisting of the field then this may suggest the likelihood of further flaring as in the case of the homologous flares studied by Ranns et al. (2000). In addition, interaction or connection with the emerging flux region behind RR could result in stressing of the magnetic field in the main already twisted structure. For example, RR's motion could in fact be produced by convective flow on unemerged parts of the single magnetic structure still below the photosphere i.e. subsurface dragging motions on the field lines connecting the two regions could be the cause of the formation of the pinch shape in the emerging flaring region. The angled direction of motion in such a scenario would result in shearing of the neutral line which we know to play an important role in flare production, especially in regions of emerging twisted magnetic flux (Kurokawa 1987). However, as mentioned previously, even a shear angle of greater than $80^{\circ}$ is not a sufficient condition to produce a flare (Chen et al. 1994). It is also known that twisting of the $\mathrm{H} \alpha$ filament structures connecting opposite polarities is an observational signature of shearing of the neutral line (Hagyard et al. 1984). The morphology of the

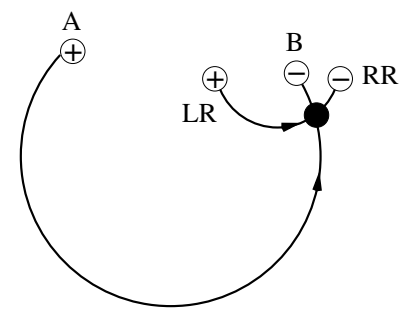

Before

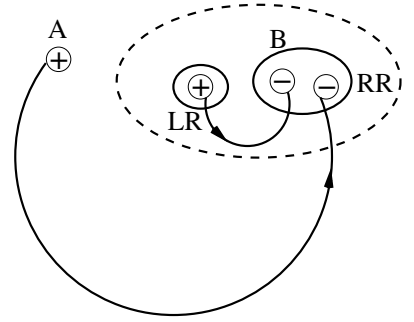

After
Fig. 17. Cartoon of emerging flux/coronal magnetic field reconnection. LR and RR correspond to the footpoints of the twisted structure shown in Fig. 12. Points A and B correspond to the footpoints of the overlying EIT loops. Partial reconnection occurs as shown in the figure The filled circle is located at the reconnection point. After reconnection some of the field lines rooted in A connect to RR and some of the field lines rooted in LR connect to B. The polarities noted from the MDI and SVST magnetograms are shown. The solid lines surrounding LR and RR/B correspond to the contour lines of the EIT brightenings (see Fig. 15). The dashed line represents the location of the SXT brightening. Note that the EIT peak intensity is situated closer to RR than LR (see text for discussion).

flare identified in this work suggests this could be occurring here. However, the angle to the center to center line is relatively small (less than $20^{\circ}$ ) so it may be that the combination of shear and emerging twisted flux is most important in this case. Note also that RR's movement, or the evolution of the magnetic structures below the photosphere, may squeeze the flux emerging parallel to the neutral line into LR. Such intrusion into an opposite polarity spot by this magnetic flux or indeed RR has been noted as a cause of shearing and flare activity in previous work (see e.g. Zirin 1983).

In the previous sect. we discussed the possibility that the emerging flux reconnects with the overlying coronal EIT loops. Figure 17 shows a cartoon picture (viewed from above) of how this might occur. Initially the twisted flux tube depicted in Fig. 16 emerges below and almost perpendicular to the overlying coronal loops. In the figure LR and RR correspond to the footpoints of the twisted structure shown in Fig. 16. Points A and B correspond to the footpoints of the overlying EIT loops. The filled circle is located at the reconnection point. The polarities noted from the MDI and SVST magnetograms are shown.

Partial magnetic reconnection then occurs in which only a small number of coronal magnetic field lines are disrupted in the manner inferred from Yohkoh observations by Hanaoka (1997) and Nishio et al. (1997). After reconnection some of the field lines rooted in A connect to RR and some of the field lines rooted in LR connect to B. Since the disruption to the magnetic field is not too catastrophic a relatively small flare is produced as a result. This is consistent with the flare observed which is of a low energy class with little associated multi-wavelength emission - we checked hard X-ray BATSE and radio data from a number of observatories. The partial reconnection occurs as shown in the figure but leaving the bulk of the field rooted in its original location so LR and RR are still connected by one loop-like (inverted S-shaped) structure. Of course continued 
emergence would disrupt the overlying field further and produce more activity at later observation times. However, we have no direct high resolution observational evidence of a change in the connectivity between magnetic elements so this scenario cannot be confirmed with the data at hand.

One further expected signature of this explanation would be the presence of a smaller intensity brightening at footpoint $\mathrm{A}$ of the larger coronal loop. To determine whether such a brightening exists we studied the intensity variations of the Fe XII $\sim 195 \AA$ images in two areas around the flare and around the other footpoint of the coronal loop. Figure 18 shows the EIT Fe XII $\sim 195 \AA$ image closest to the flare peak time at 14:51 UT with the two chosen areas overlaid. It also shows the time profiles of the total intensities (arbitrary units) in these areas. There is a clear indication of a weaker brightening in Area 2 at the peak flare time which is additional evidence in support of this model. In order to confirm that the brightening was significant and not a result of instrumental effects, we examined the intensity variations in control regions around Areas 1 and 2. We selected the 8 regions adjacent to Area 1 and the 8 regions adjacent to Area 2. All the regions were taken to be the same size. We totalled the intensities in these regions and normalised them by the peak intensity. We then compared the peak intensity to the average of the preceding 3 exposures. Thus the flare intensity increased by $\sim 33 \%$ and the intensity in Area 2 increased by $\sim 15 \%$. These two areas recorded the largest intensity increases. Of the 16 control regions considered, 6 overlapped significantly and so we discarded the 3 that showed the smallest intensity changes. In the remaining 13 cases, 9 showed increases and 4 showed decreases. In only one case was the intensity increase greater than $8 \%$. This region is marked in Fig. 18 by dotted lines and the intensity increase was $10.5 \%$. Interestingly it appears to be positioned further along the loop between the footpoint brightenings. This analysis indicates that the distant footpoint brightening in Area 2 is real. We note also that if the flare is caused by reconnection with the coronal field, then since both regions emerge below the same magnetic loop, the twisted and sheared region must be more susceptible to changing its magnetic configuration. The convergence of anti-parallel fields alone is not sufficient to cause flaring.

The solid lines in Fig. 17 surrounding LR and RR/B correspond to the contour lines of the most intense EIT brightenings (see Fig. 15). The EIT image actually shows a loop-like brightening the footpoints of which we are not able to resolve from the images (see Fig. 1 upper left panel and the lowest level contour in Fig. 14). Only when we identify the absolute peak intensities do we see that RR is brighter than LR (see Fig. 14 and the highest level contour in Fig. 15). The SXT image shows a brightening covering the whole region and this is indicated by the dashed line in the figure The uniform brightening in the SXT image comes from the top of the reconnected loops. As mentioned the EIT brightening is also fairly uniform along the large coronal loop leg and comes from within the reconnected loops. However, the most intense EIT brightenings can be localised further to positions near the loop footpoints (as viewed from above). In our scenario the EIT peak intensity is situated above RR as a result of observing the combined intensity of the
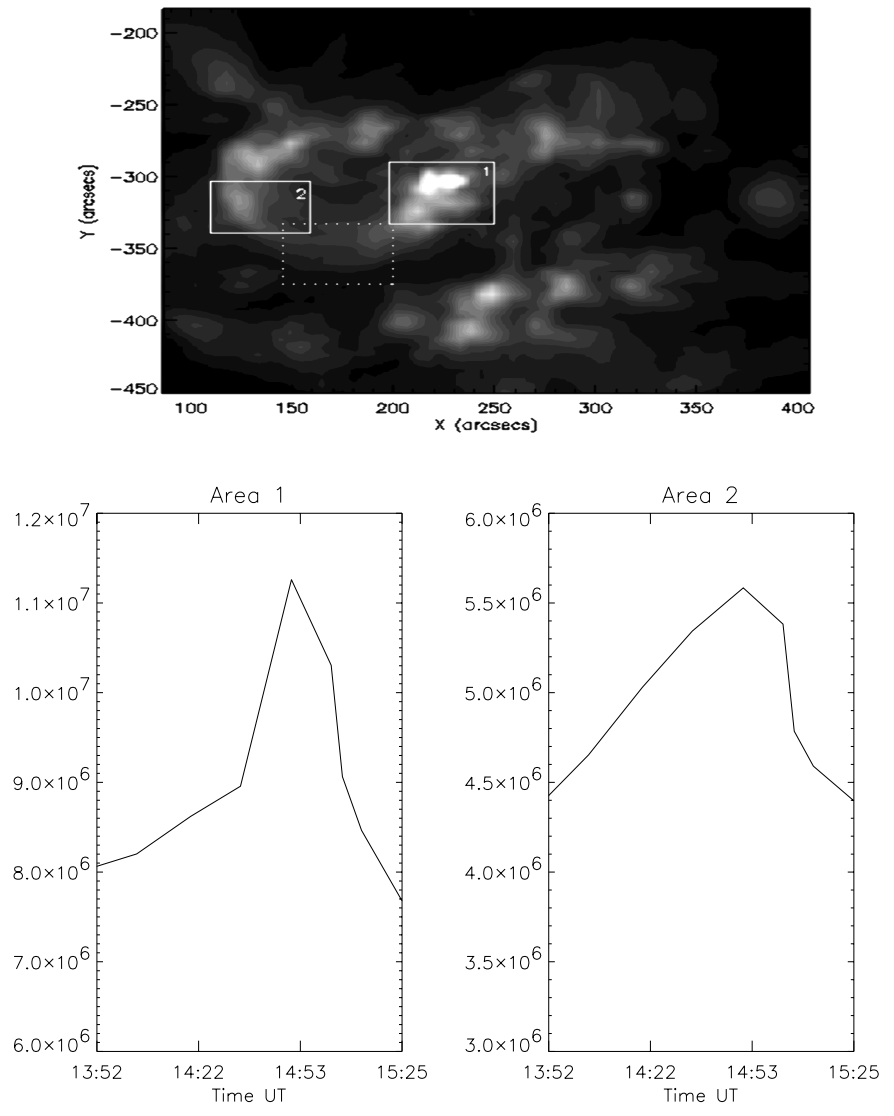

Fig. 18. Evidence for brightenings at the coronal loop footpoints. Upper panel: EIT $195 \AA$ image taken at 14:51 UT. Area 1 is around the flare region and Area 2 is around the other footpoint of the coronal loop. The dotted region marks the control area with the most significant brightening (see text). Lower panel: Time profiles of the intensities in Areas 1 and 2.

footpoints of two reconnected loops rather than just one - as in the case of LR.

We also searched for direct observational evidence of the flare trigger mechanism. Fletcher et al. (2001) recently analysed a flare event in 1993 May and suggested that the flare was caused by disruption of the coronal magnetic field by smallscale flux cancellation at the photosphere between strong opposite magnetic polarities. Such cancellations may manifest themselves as small scale brightenings in EUV images (e.g. from TRACE) near the interface of opposite polarity magnetic elements. Our data certainly show pervasive changes in the small-scale photospheric magnetic field together with numerous brightenings in the $\mathrm{H} \alpha$ dataset. These brightenings are also noticeable in the light curves and are presumably located closer to the photosphere (and hence the magnetic flux cancellation points) than the EUV brightenings. However, we were unable to identify a specific magnetic mergence or series of brightenings which caused the flare although we did identify the attachment and separation of the $\mathrm{H} \alpha$ dark structures which may be indirectly related. It would be interesting to compare simultaneous $\mathrm{H} \alpha$ and EUV pre-flare brightenings.

Finally, we note the similarity between the inverted $\mathrm{S}$-shaped structure which forms prior to the occurrence of the 
small flare and the morphology of soft X-ray "sigmoid" active regions (Canfield et al. 1999). Canfield et al. (1999) found a strong relationship between active regions classified as sigmoid, and their likelihood of eruption. It is interesting that on a much smaller scale at lower temperatures the inverted S-shaped structure is associated with a flare, while the arch filament in region $\mathrm{B}$ is not.

\section{Conclusions}

We have studied cotemporal observations of a small two-ribbon flare observed in active region NOAA 8218 on 1998, May 13th. The data analysed were recorded by the SVST, formerly located on the island of La Palma, Spain, in the $4305 \AA$ $G$-band, the $\mathrm{H} \alpha 6356 \AA$ line center and red and blue wings at $+/-350 \mathrm{m \AA}$ and +/- $700 \mathrm{~m} \AA$. Simultaneous Fe I $6302 \AA$ images were obtained to produce magnetograms. The results were also compared to full-disk SOHO-MDI magnetograms and SOHO-EIT images.

Our principle aim was to use simultaneous multiwavelength coverage to study the causal relationship between emerging flux and solar flare production in a comparatively simple geometry throughout the flare duration. The outstanding spatial resolution capabilities of the SVST (close to 0.3") allow us to examine the evolution of the flare in greater detail than before. A further objective was to compare and contrast the flare region with a non-flaring emerging flux region within the same field of view and analyse the differences between them to assess the essential processes involved and the flare trigger mechanism.

Analysis of the temporal evolution of magnetic flux and a geometrical interpretation of feature tracking and correlation analysis results confirm that both regions are associated with emerging flux. Unusual sunspot motions, coincident with the $\mathrm{H} \alpha$ flare kernels, are observed which result in a high level of shearing of the magnetic neutral line between the spots. In contrast the non-flaring region shows separating footpoint motions, with apparent arch-like filament connections in the $\mathrm{H} \alpha$ data, consistent with the usual appearance of emerging flux. We interpret the motions as evidence of additional twisting of the emerging flux tube in the flaring region compared to the non-flaring region. New magnetic flux emergence perpendicular to the overlying field indicates the emergence of a strongly twisted magnetic flux rope which developed a sheared configuration with the overlying magnetic field as evidenced by the $\mathrm{H} \alpha$ inverted S-shaped feature. If so our results confirm, with higher resolution observational data, the conclusions of previous authors that the emergence of twisted magnetic flux plays an important role in flare production in regions of magnetic shear (Kurokawa 1987; Tanaka 1991; Ishii et al. 1998; Li et al. 2000). The results also confirm that flares do not occur if opposite polarities are connected by simple arches perpendicular to the neutral line (Zirin 1983), even a small twist can make the difference.

We also examined the dark inverted S-shaped feature in the $\mathrm{H} \alpha$ images which showed considerable restructuring during the observing period (see Fig. 4). These structures appear to attach prior to the flare and then separate after the flare energy release disrupts the magnetic field. This implies that the attachment of the structures may be an observational consequence of the magnetic reconnection or untwisting of the field which triggered the flare. We also find evidence that the build-up of magnetic flux is faster in the flaring region than in the non-flaring region and that the EIT peak intensity is located above the footpoint of greater magnetic field strength.

We proposed a simple schematic model of the emergence of a twisted flux tube in an $\Omega$ shape with one straight "trunk" and a strongly twisted attached "branch-like" structure between the trunks. This model can account for the observational details such as the footpoint motions, elongated thread-like sunspots perpendicular to the overlying field and $\mathrm{H} \alpha$ structures of the flaring region. We also discussed ways in which such a model can, in principle, trigger a flare by partial reconnection or untwisting of the magnetic field. We found some evidence of brightenings at the coronal loop footpoints which may support the former scenario. However, we were unable to detect the flare trigger or definitively confirm the model with the observational data available.

Our study also reveals the direction for future work of this type. Although the magnetic morphology of the flare appeared quite simple and the observations were of the highest quality, it is clear that extended dedicated high resolution multi-wavelength observations over long pre- and post- flare timescales of similar low energy flares are needed due to the actual complexity even of this small flare. Pre-flare $G$-band and high resolution co-pointed EUV images would confirm the connectivity between the flare footpoints and their observed motion from the emerging magnetic configuration. Post-flare observations would identify any repetitive behaviour or further disruption to the coronal magnetic field which is important for comparison with theoretical models. Such topics can be addressed with future coordinated multi-wavelength observations from ground and space based observatories.

Acknowledgements. The authors would like to thank H. Aurass (Astrophysikalisches Institut Potsdam, Germany), S. Zieba (Jagiellonian University, Poland) and A. Kerdraon (Observatoire de Paris, France) for providing radio data for the date of our observations and A. Csillagy and P. Messmer for help with the ETH Zurich archive. We also thank J. Ryan (Univ. of New Hampshire), C. Schrader and D. Biesecker (NASA-GSFC) for help in obtaining Compton Gamma Ray Observatory BATSE data. We would also like to thank D. Biesecker again and P. F. Chen, T. Morimoto, T. T. Ishii and A. Asai for useful discussions. We thank the EIT and MDI consortia for providing data. The SVST is operated by the Swedish Royal Academy of Sciences. The Observatorio del Roque de los Muchachos is run by the Spanish Instituto de Astrofísica de Canarias on the island of La Palma. Yohkoh is a mission of the Institute of Space and Astronautical Sciences (Japan), with participation from the US and UK. SOHO is a mission of international cooperation between ESA and NASA. One of the authors (H. Kurokawa) would like to thank Dr. Allan Title for providing the La Palma data and for his invitation to Lockheed, Palo Alto. Finally, we would like to thank the anonymous referee for many good suggestions that helped to improve the manuscript. 


\section{References}

Aschwanden, M. J., Kosugi, T., Yoichiro, H., Nishio, M., \& Melrose, D. B. 1999, ApJ, 526, 1026

Berger, T. E., \& Lites, B. W. 2002, Sol. Phys., 208, 181

Berger, T. E., \& Title, A. M. 2001, ApJ, 553, 449

Canfield, R. C., Hudson, H. S., \& McKenzie, D. E. 1999, Geophys. Res. Lett., 26, 6, 627

Chen, J., Wang, H., Zirin, H., \& Ai, G. 1994, Sol. Phys., 154, 261

Delaboudiniére, J.-P., Artzner, G. E., Brunaud, J., et al. 1995, Sol. Phys., 162, 291

Fletcher, L., Metcalf, T. R., Alexander, D., Brown, D. S., \& Ryder, L. A. 2001, 554, 415

Forbes, T. G., \& Priest, E. R. 1984, Sol. Phys., 94, 315

Hagyard, M. J., Smith, J. B., Jr., Teuber, D., \& West, E. A. 1984, Sol. Phys., 91, 115

Hara, H., Tsuneta, S., Lemen, J. R., Acton, L. W., \& McTiernan, J. M. 1992, PASJ, 44, L135

Hanaoka, Y. 1997, Sol. Phys., 173,319

Heyvaerts, J., Priest, E. R., \& Rust, D. M. 1977, ApJ, 216, 123

Ishii, T. T., Kurokawa, H., \& Takeuchi, T. T. 1998, ApJ, 499, 898

Ishii, T. T., Kurokawa, H., \& Takeuchi, T. T. 2000, PASJ, 52, 337

Jähne, B. 1997, in Practical Handbook in Image Processing for Science Applications, Chap. 8 (Boca Raton: CRC Press)

Kamio, S., Kurokawa, H., \& Ishii, T. T. 2003, Sol. Phys., in press

Kurokawa, H. 1987, Sol. Phys., 113, 259
Kurokawa, H. 1991, in Lecture Notes in Physics, ed Y. Uchida, R. C. Canfield, T. Watanabe, \& E. Hiei (Berlin, Heidelberg, New York: Springer-Verlag), 387, 39

Kurokawa, H. 1996, in MHD Phenomena in the Solar Atmosphere, Prototypes of Stellar Magnetic Activity, ed. Y. Uchida, T. Kosugi, \& H. S. Hudson (Dordrecht: Kluwer), 185

Kurokawa, H., Wang, T., \& Ishii, T. T. 2002, ApJ, 572, 598

Li, H., Sakurai, T., Ichimoto, K., \& Ueno, S. 2000, PASJ, 52,465

Linton, M. G., Dahlburg, R. B., \& Antiochos, S. K. 2001, ApJ, 553, 905

Nishio, M., Yaji, K., Kosugi, T., Nakajima, H., \& Sakurai, T. 1997, ApJ, 489, 976

Piddington, J. H. 1974, Sol. Phys., 38, 465,

Ranns, N. D. R., Harra, L. K., Matthews, S. A., \& Culhane, J. L. 2000, 360,1163

Scharmer, G. B., \& Lofdahl, M. 1991, Adv. Spa. Res., 11, 129

Scharmer, G. B., Pettersson, L., Brown, D. S., \& Rehn, J. 1985, App. Opt., 24, 2558

Scherrer, P. H., Bogart, R. S., Bush, R. I., et al. 1995, Sol. Phys., 162, 129

Shibata, K., \& Yokoyama, T. 2002, ApJ, 577, 422

Shibata, K., Nozawa, S., \& Matsumoto, R. 1992, PASJ, 44, 265

Stein, R., \& Nordlund, A. 2002, in Proc. of IAU Colloq., 188, in press Tanaka, K. 1991, Sol. Phys., 136, 133

Van Driel-Gesztelyi, L., et al. 1996, Sol. Phys., 163, 145

Zirin, H. 1983, ApJ, 274, 900

Zirin, H., \& Tanaka, K. 1973, Sol. Phys., 32, 173 